\title{
VARIABILITÉ INFRASPÉCIFIQUE DE L'ÉPICÉA (PICEA ABIES KARST., PARTIE SEPTENTRIONALE DE L'AIRE FRANÇAISE). HÉRITABILITÉ ET CORRÉLATIONS GÉNÉTIQUES DE QUELQUES CARACTÈRES AU STADE JUVÉNILE
}

\author{
J.-F. LACAZE et M. ARBEZ \\ Station d'Amélioration des Arbres forestiers, \\ Centre national de Recherches forestières, I.N.R.A., \\ 54 - Nancy
}

\section{RÉSUMÉ}

Cet article fait le bilan d'une expérimentation concernant quinze provenances naturelles de la partie septentrionale de l'aire française de l'épicéa (Picea abies Karst.), chaque provenance étant représentée par dix descendances maternelles. Les observations et mesures ont été effectuées sur de jeunes plants (trois et quatre ans).

Les résultats concernant la variabilité inter-provenances confirment ceux d'expériences antérieures. Ils se résument comme suit :

- Vosges (Gérardmer) : forte vigueur, précocité du débourrement végétatif marquée, fréquence élevée de pousses daoût, faible proportion d'individus à aiguilles glauques, densité du bois faible.

- Deuxième plateau du Jura et Préalpes d'altitude: vigueur moyenne, débourrement tardif (sauf Mignovillard), fréquence moyenne de pousses d'août, assez faible proportion d'individus à aiguilles glauques, densité du bois moyenne.

- Jura, hautes chaînes et Préalpes de haute altitude : vigueur faible, débourrement précoce, peu de pousses d'août, fréquence élevée d'individus à aiguilles glauques, forte densité du bois.

La variabilité intra-provenance est examinée sous les deux aspects inter-familles et intrafamille ou résiduelle.

La variabilité inter-familles exprime, à quelques réserves près, la variance génétique additive des mères. Elle atteint ici un niveau élevé pour les caractères mesurés (vigueur et phénologie). On remarque que l'utilisation de quelques descendances d'arbres \& plus s ne semble pas apporter de biais; par contre, l'effectif des mères par provenance (dix) se révèle nettement insuffisant (erreur d'échantillonnage, écart à la normalité). L'amplitude de cette variabilité inter-familles varie notablement avec les provenances. Pour la vigueur, elle atteint le niveau le plus élevé chez les provenances de haute altitude. 
La variabilité résiduelle exprime des effets divers (effets génétiques, additifs ou non ; effets de milieu). Celle-ci diffère également selon les provenances, mais ici les écarts s'expliquent surtout par des *effets d'échelle *.

L'examen de la répartition de la variabilité entre les différents niveaux montre que:

- Pour la hauteur, la variance résiduelle (incontrôlable) représente approximativement la moitié de la variance totale: la part de variabilité due à leffet provenance (35\%) est ici plus importante que celle ressortant de la variabilité individuelle $(4 \times 5,6 \%)$.

- Pour le débourrement, la variance résiduelle est encore plus élevée $(78 \%)$, mais lamplitude de la variabilité semble plus forte au niveau individuel qu'au niveau provenance.

Il apparaît done que la sélection de provenances garde tout son intérêt pour l'amélioration de la vigueur, de même que la sélection individuelle pour lobtention de matériel tardif.

Une classification objective (analyse des composantes principales) des provenances permet de procéder aux regroupements logiques suivants:

- Groupe I: cinq provenances d'altitude du deuxième Plateau du Jura et des Préalpes,

- Groupe II : sept provenances de haute altitude du Jura et des Préalpes.

- Provenance Gérardmer (Vosges) : moyenne altitude.

A l'intérieur des groupes I et II. les différences entre provenances sont assez réduites pour que l'on puisse considérer l'ensemble des familles correspondantes comme faisant partie de deux populations théoriques homogènes d'où l'avantage de réduire l'erreur d'échantillonnage due à un effectif de familles trop faible.

L'héritabilité de divers caractères a été calculée pour les trois critères définis précédemment. L'héritabilité de la vigueur est notablement plus élevée pour le groupe II que pour le groupe $I$ et Gérardmer. Les interactions entre gènes joueraient un rôle moins important dans les pessières d'altitude (niveau d'hétérozygotie moins élevé). L'héritabilité des caractères phénologiques est voisine pour les deux grands groupes.

Les corrélations génétiques additives entre mesures de dimension ou poids dorganes sont très élevées. Vigueur et pousse d'août se relient positivement, vigueur et couleur glauque négativement. Pour le couple vigueur-précocité du débourrement, la liaison additive est soit négative (groupe II), soit nulle (groupe I). La corrélation due à lenvironnement est négative dans les deux cas.

Ces quelques résultats prouvent que corrélations génétiques et héritabilités sont des paramètres caractéristiques de populations et non seulement d'espèces, et que les programmes de sélection individuelle devront être élaborés au niveau des provenances ou groupes de provenances.

\section{1. - INTRODUCTION}

Les reboiseurs attachent une importance croissante au choix des sources de graines, c'est-à-dire à la sélection des provenances. Ce problème a été abordé à diverses reprises dans cette revue.

Le stade ultérieur de l'amélioration génétique consiste à sélectionner dans ces populations, génétiquement supérieures, des individus également supérieurs à la moyenne pour un certain nombre de caractères, puis à les croiser entre eux pour obtenir des graines d'une variété synthétique qui sera mise à la disposition des praticiens.

L'efficacité de la sélection individuelle qui se traduit concrètement par le gain génétique inscrit dans la variété synthétique dépend essentiellement des paramètres suivants :

— intensité de sélection (proportion d'individus sélectionnés par rapport à l'effectif total) ;

- variabilité individuelle au sein de la population choisie (variance intrapopulation) ; 
— part de l'écart individuel transmissible à ses descendants (héritabilité) ;

- liaison génétique entre caractères: une sélection sur l'un d'entre eux peut entraîner une sélection indirecte favorable ou défavorable sur les autres.

La liste des caractères retenus est établie par les utilisateurs en fonction de données économiques ; le chercheur est généralement maitre du premier paramètre (peuplements à effectifs généralement importants), mais il doit obtenir des informations sur les trois autres avant d'engager le programme de sélection proprement dit.

L'expérimentation dont il va être rendu compte a pour objectif d'obtenir une estimation de ces trois données fondamentales: variabilité, héritabilité, corrélations génétiques, pour quelques caractères importants chez l'épicéa (Picea abies).

\section{2. - ORGANISATION DE L'EXPÉRIENCE}

\section{1. - Matériel végétal}

L'expérience comprend 15 provenances naturelles représentant de façon assez satisfaisante la variabilité de l'espèce dans la partie septentrionale de laire française (Jura, Vosges, Préalpes du Nord). Pour la plupart, ces provenances avaient déjà été étudiées dans les précédentes expérimentations françaises (BovVAREI, 1962; LACAZE, 1969).

Chaque provenance est représentée par les descendances maternelles (pollinisation libre) de 10 arbres. Dans la plupart des cas, ces arbres ont été choisis au hasard dans l'étage dominant, et ils étaient distants de plus de $50 \mathrm{~m}$ les uns des autres. Toutefois, certaines provenances (Gérardmer, Esserval-Tartre, Bonnétage, Grande-Chartreuse) sont représentées par une majorité d'arbres s plus s (11). Le tableau 1 et la figure 1 apportent de plus amples informations sur les provenances et leur composition.

\section{2. - Dispositif en pépinière}

Les descendances maternelles ont été semées en plein, sans dispositif, au printemps 1963. A l'âge de deux ans, à lautomne 1965 , ces semis furent repiqués à l'écartement de $12,5 \times$ $12,5 \mathrm{~cm}\left(64\right.$ plants $\left./ \mathrm{m}^{2}\right)$, selon le dispositif statistique suivant:

Blocs incomplets équilibrés avec split-plot :

- 15 provenances, 7 répétitions, 35 blocs;

3 provenances par bloc:

- varcelles unitaires de 160 individus (provenance) comportant 10 sous-parcelles (split-plot), de 16 individus (descendances dans provenance).

\section{3. - Mesures et observations}

Les mesures et observations font robjet du tableau 2.

Les caractères mesurés (quantitatifs) ou observés (qualitatifs), au cours des $3^{*}$ et $4^{*}$ année de végétation, se rattachent à quatre grands groupes:

- Production $=$ dimensions (hauteur et diamètre) et poids secs.

- Qualité du bois (densité).

- Phénologie (débourrement, pousse d’août).

- Morphologie (couleur et insertion des aiguilles).

Excepté le diamètre au collet et les poids secs portant sur un échantillon plus restreint. les autres caractères furent mesurés ou observés sur tous les individus du dispositif soit en principe 112 par descendance (1.120 par provenance).

(1) Sélection phénotypique individuelle, pour la forme d'une part, la production d'autre part, mais avec, a priori, une efficacité faible dans ce dernier cas, 


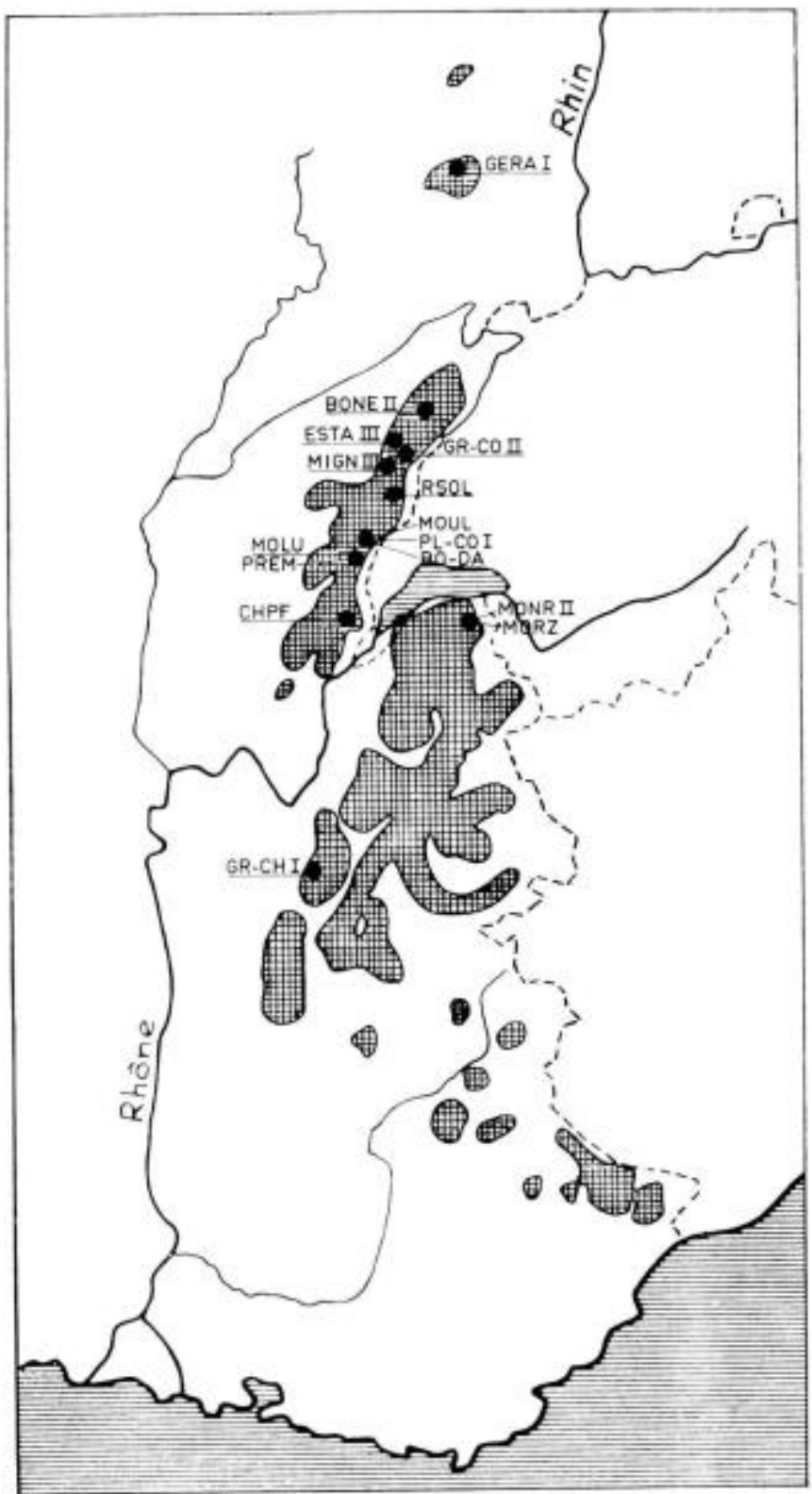

FIG. 1. - Aire française de l'Epicéa. Plovenances étudiées. FiG. 1. - French area of spruce. Sudied provenances 
Tableau 1 - table 1

Description des provenances

Description of provenances

\begin{tabular}{|c|c|c|c|c|c|c|c|c|}
\hline Nom & $\begin{array}{l}\text { Abrévia- } \\
\text { tion }\end{array}$ & Région & $\begin{array}{l}\text { Longitude } \\
\text { est }\end{array}$ & $\begin{array}{l}\text { Latitude } \\
\text { nord }\end{array}$ & $\underset{(\mathrm{m})}{\text { Altitude }}$ & $\begin{array}{c}\text { Pluvio- } \\
\text { métrie } \\
(\mathrm{mm})\end{array}$ & Roche-mère & Arbres \& + \\
\hline Grande-Chartreuse I .... & GR-CH I & $\begin{array}{l}\text { Préalpes du Nord, } \\
\text { altitude }\end{array}$ & $5^{\circ} 45^{\prime}$ & $45^{\circ} 22^{\prime}$ & $750-840$ & 1.800 & $\begin{array}{l}\text { Marnes du valan- } \\
\text { ginien moyen }\end{array}$ & $\begin{array}{l}\text { CTR 1, 2, 3, } 4,5, \\
6,7,8,9, \text { PMO } 6 .\end{array}$ \\
\hline Montriond 11 ........... & MONR II & $\begin{array}{l}\text { Préalpes du Nord, } \\
\text { altitude }\end{array}$ & $6^{\circ} 44^{\prime}$ & $46^{\circ} 12^{\prime}$ & $1.100-1.200$ & 1.406 & Brèche calcaire & MRI 1. \\
\hline Morzine & MORZ & $\begin{array}{l}\text { Préalpes du Nord, } \\
\text { haute altitude }\end{array}$ & $6^{\circ} 48^{\circ}$ & $46^{\circ} 12^{\prime}$ & $1.700-1.800$ & 1.406 & & \\
\hline Champfromier ... & CHPF & $\begin{array}{l}\text { Jura, hautes chai- } \\
\text { nes }\end{array}$ & $5^{\circ} 52^{\circ}$ & $46^{\circ} 13^{\circ}$ & $1.160-1.180$ & 1.391 & $\begin{array}{l}\text { Calcaire jurassique } \\
\text { superficiel tendant } \\
\text { au lapiaz }\end{array}$ & \\
\hline Molunes ................. & MOLU & $\begin{array}{l}\text { Jura, hautes chai- } \\
\text { nes }\end{array}$ & $6^{\circ} 04^{\circ}$ & $46^{\circ} 25^{\prime}$ & $1.340-1.380$ & 1.531 & $\begin{array}{l}\text { Calcaire jurassique } \\
\text { supérieur }\end{array}$ & \\
\hline Prémanon..... & PREM & $\begin{array}{l}\text { Jura, hautes chai- } \\
\text { nes }\end{array}$ & $6^{\circ} 04^{+}$ & $46^{\circ} 25^{\prime}$ & $1.340-1.380$ & 1.531 & $=$ & \\
\hline Plan des Cosaques 1 .... & PL-CO 1 & $\begin{array}{l}\text { Jura, hautes chai- } \\
\text { nes }\end{array}$ & $6^{\circ} 06^{\circ}$ & $46^{\circ} 29^{\circ}$ & 1.170 & 1.663 & , & \\
\hline Bois d'Amont......... & BO-DA & $\begin{array}{l}\text { Jura, hautes chai- } \\
\text { nes }\end{array}$ & $6^{\circ} 06^{\circ}$ & $46^{\circ} 29^{\circ}$ & 1.180 & 1.663 & $\begin{array}{l}\text { Calcaire jurassique } \\
\text { supérieur }\end{array}$ & RIS 4. \\
\hline La Mouille ......... & MOUL & $\begin{array}{l}\text { Jura, hautes chai- } \\
\text { nes }\end{array}$ & $6^{\circ} 06^{\circ}$ & $46^{\circ} 29^{\circ}$ & 1.200 & 1.663 & $\rightarrow$ & \\
\hline Risol ............. & RSOL & $\begin{array}{l}\text { Jura, hautes chaî- } \\
\text { nes }\end{array}$ & $6^{\prime \prime} 14^{\prime}$ & $46^{\circ} 38^{\prime}$ & $1.230-1.380$ & 1.602 & $\begin{array}{l}\text { Calcaire kimme } \\
\text { ridgien }\end{array}$ & \\
\hline Mignovillard III $\ldots \ldots \ldots$. & MIGN HI & Jura, $2^{\circ}$ plateau & $6^{*} 12^{\prime}$ & $46^{\circ} 45^{\prime}$ & $1.000-1.100$ & 1.602 & $\begin{array}{l}\text { Calcaire jurassique } \\
\text { supérieut }\end{array}$ & \\
\hline Grand-Côte II ............ & GR-CO II & Jura, $2^{*}$ plateau & $6^{*} 16$ & $46^{\circ} 46^{\circ}$ & 950 & 1.550 & $\begin{array}{l}\text { Calcaire dur port- } \\
\text { landien }\end{array}$ & \\
\hline Esserval-Tartre III $\ldots . .$. . & ES-TA III & Jura, $2^{*}$ plateau & $6^{*} 05^{\prime}$ & $46^{\circ} 50^{\circ}$ & 800 & 1,663 & $\begin{array}{l}\text { Calcaire jurassique } \\
\text { supérieur }\end{array}$ & $\begin{array}{l}\text { EST } 4,5,6,7,8, \\
9,10 .\end{array}$ \\
\hline Bonnétage II ,.... & BONE II & Jura, $2^{2}$ plateau & $6^{\circ} 45^{\circ}$ & $47^{\circ} 10^{\circ}$ & 875 & 1.390 & Calcaire & $\begin{array}{l}\text { BON } 1,3,4,5,6 \\
7,8,9 .\end{array}$ \\
\hline Gérardmer $1, \ldots \ldots \ldots . .$. & GERA 1 & Vosges centrales & $6^{\circ} 54^{\circ}$ & $48^{\circ} 11^{\prime}$ & $650-750$ & 1.530 & $\begin{array}{l}\text { Eboulis graniti- } \\
\text { ques }\end{array}$ & $\begin{array}{l}\text { GER } 3,4,5,6,13, \\
14,15,18,19,27 .\end{array}$ \\
\hline
\end{tabular}


TABLEAU 2 - TABLE 2

Description des caractères étudiés

Description of studied characters

\begin{tabular}{|c|c|c|c|c|}
\hline $\begin{array}{l}\text { Numéro } \\
\text { du } \\
\text { caractère } \\
\text { [Number } \\
\text { of the } \\
\text { character] }\end{array}$ & $\begin{array}{c}\text { Description du caractère } \\
\text { [Description of the character] }\end{array}$ & $\begin{array}{l}\text { Echelle de notation ou précision } \\
\text { des mesures individuelles }\end{array}$ & $\begin{array}{l}\text { Nombre total } \\
\text { théorique et réel } \\
\text { (entre parenthèses) } \\
\text { d'individus mesurés } \\
\text { par descendance }\end{array}$ & $\begin{array}{l}\text { Date de mesure } \\
\text { ou observation }\end{array}$ \\
\hline $1 \ldots \ldots$ & $\begin{array}{l}\text { Hauteur totale aे } 3 \text { ans } \\
\text { [Total height at } 3 \text { years] }\end{array}$ & $0,5 \mathrm{~cm}$ & $112(102)$ & $-/ 1 / 67$ \\
\hline $2 \ldots \ldots$ & $\begin{array}{l}\text { Hauteur totale à } 4 \text { ans } \\
\text { [Total height at } 4 \text { years] }\end{array}$ & $1 \mathrm{~cm}$ & $112(102)$ & $-/ 12 / 67$ \\
\hline $3 \ldots \ldots$ & $\begin{array}{l}\text { Diamètre au collet, à } 4 \text { ans (1) } \\
\text { [Collar diameter at } 4 \text { years] }\end{array}$ & $1 / 10^{\circ} \mathrm{mm}$ & $80(68)(1)$ & $-/ 12 / 67$ \\
\hline \multirow[t]{2}{*}{$4 \ldots \ldots$} & $\begin{array}{l}\text { Poids sec de tige, à } 4 \text { ans } \\
\text { [Stem dry weight at } 4 \text { years] }\end{array}$ & $1 / 10^{\circ} \mathrm{mg}$ & $10(6,85)(2)$ & \\
\hline & $\begin{array}{l}\text { Poids sec de branches, à } 4 \text { ans } \\
\text { [Branch dry weight at } 4 \text { years] }\end{array}$ & $1 / 10^{\circ} \mathrm{mg}$ & $10(6,85)(2)$ & \\
\hline \multirow[t]{2}{*}{$5 \ldots \ldots$} & $\begin{array}{l}\text { Poids sec d'aiguilles, à } 4 \text { ans } \\
\text { [Nezdle dry weight at } 4 \text { years] }\end{array}$ & $1 / 10 \% \mathrm{mg}$ & $10(6,85)(2)$ & \\
\hline & $\begin{array}{l}\text { Poids sec de racines, à } 4 \text { ans } \\
\text { [Root dry weight at } 4 \text { years] }\end{array}$ & $1 / 10 \mathrm{mg}$ & $10(6,85)(2)$ & \\
\hline \multirow[t]{2}{*}{$6 \ldots \ldots$} & $\begin{array}{l}\text { Densité du bois de tige, à } 4 \text { ans } \\
\text { [Stem wood density at } 4 \text { years] }\end{array}$ & $10-3$ & $10(6,85)(2)$ & \\
\hline & $\begin{array}{l}\text { Débourrement de } 4^{*} \text { année (1 }{ }^{\text {re }} \text { observation) } \\
\text { [Flushing on the } 4 \text { th year (1st observation)] }\end{array}$ & $\begin{array}{c}\text { Note: } 0 \text {, bourgeon non débourré (1) } \\
1 \text {, bourgeon débourré }\end{array}$ & $112(102)$ & $20.22 / 4 / 67$ (4) \\
\hline $7 \ldots \ldots$ & $\begin{array}{l}\text { Débourrement } 4^{*} \text { année }\left(2^{*} \text { observation) }\right. \\
\text { [Flushing on the } 4 \text { th year (2nd observation)] }\end{array}$ & $d^{\prime \prime}$ & $112(102)$ & $27-29 / 4 / 67$ \\
\hline
\end{tabular}




\begin{tabular}{|c|c|c|c|c|c|}
\hline & & $\begin{array}{l}\text { Débourrement } 4^{*} \text { année }\left(3^{*} \text { observation) }\right. \\
\text { [Flushing on the } 4 \text { th year (3nd observation)] }\end{array}$ & $d^{\prime \prime}$ & $112(102)$ & $5-6 / 5 / 67$ \\
\hline & & $\begin{array}{l}\text { Débourrement } 4^{*} \text { année }\left(4^{*} \text { observation) }\right. \\
\text { [Flushing on the } 4 \text { th year ( } 4 \text { th observation)] }\end{array}$ & $d^{\prime \prime}$ & $112(102)$ & $12-13 / 5 / 67$ \\
\hline 8 & $\ldots \ldots$ & $\begin{array}{l}\text { Somme des } 4 \text { notes individuelles de débour- } \\
\text { rement de } 4^{*} \text { année } \\
\text { [Sum of the } 4 \text { individuals flushing notations } \\
\text { on the } 4 \text { th year] }\end{array}$ & & $112(102)$ & \\
\hline 9 & $\cdots \cdots$ & $\begin{array}{l}\text { Dégâts de gelée de printemps (4* année) } \\
\text { [Spring frost damages (4th year)] }\end{array}$ & $\begin{aligned} & \text { Note: } 0 \text {, aucun dégât } \\
& 1 \text {, dégất sur bourgeons latéraux } \\
& 2 \text {, dégất sur bourgeon terminal }\end{aligned}$ & $112(102)$ & $5 / 6 / 67$ \\
\hline 10 & $\ldots \ldots$ & $\begin{array}{l}\text { Pousse d'août de } 3^{*} \text { année } \\
\text { [Lamma shoot on the 3rd year] }\end{array}$ & $\begin{array}{l}\text { Note : } 0 \text {, aucune pousse d'août } \\
1 \text {, pousse d'août sur bourgeons laté- } \\
\text { raux seuls } \\
2 \text {, pousse d'août sur bourgeon termi- } \\
\text { nal (et éventuellement bourgeons latéraux) } \\
3 \text {, élongation continue de la pousse } \\
\text { terminale }\end{array}$ & $112(102)$ & $10-18 / 8 / 66$ \\
\hline 11 & $\ldots \ldots$ & $\begin{array}{l}\text { Pousse d'aout de } 4^{e} \text { année } \\
\text { [Lamma shoot on the } 4 \text { th year] }\end{array}$ & Idem à pousse d'août de $3^{*}$ année & $112(102)$ & $3-5 / 8 / 67$ \\
\hline 12 & … & $\begin{array}{l}\text { Couleur des aiguilles à } 4 \text { ans } \\
\text { [Needle color at } 4 \text { years] }\end{array}$ & $\begin{array}{r}\text { Note: } 0 \text {, vert franc } \\
1 \text {, vert bleu }\end{array}$ & $112(102)$ & $30 / 8$ au $5 / 9 / 67$ \\
\hline 13 & $\cdots \cdots$ & $\begin{array}{l}\text { Insertion des aiguilles sur la pousse termi- } \\
\text { nale de } 4^{\circ} \text { année } \\
\text { [Insertion of the needles on the leader shoot } \\
\text { (4th year)] }\end{array}$ & $\begin{array}{l}\text { Note : } 0 \text {, aiguilles appliquées } \\
\text { 1, aiguilles écartées } \\
\text { 2, aiguilles très écartées } \\
\text { 3, aiguilles rabattues }\end{array}$ & $112(102)$ & $-/ 12 / 67$ \\
\hline
\end{tabular}

(1) Mesure effectuée sur 5 répétitions du dispositif seulement.

(2) Mesure sur 2 individus par descendance et par répétition sur les 3 premières répétitions et 1 individu seulement sur les 4 dernières, choisis de telle façon que leur hauteur totale à 4 ans soit très voisine de celle de la moyenne de la sous-parcelle à laquelle ils appartiennent.

(3) Le nombre total d'individus à observer et le personnel disponible ne permettait pas de terminer les observations en une seule journée. Toutefois, tous les individus appartenant à la méme répétition (done a fortiori au méme bloc) étaient observés dans la méme journéc.

(4) Concernant les caractères qualitatifs, on a utilisé suivant les cas, soit les notations individuelles sans transformation (caractères 7, 8, 9, 10, $11,12,13$ ), soit le pourcentage de note 1 (earactère 7,12 ) après transformation par la fonction arc sin. $V$. 


\section{3. - VARIABILITÉS INTER-PROVENANCES ET INTRA-PROVENANCE (INDIVIDUELLE), VARIABILITÉ RÉSIDUELLE}

\section{1. - Variabilité inter-provenances}

Le tableau 3 renseigne sur la variabilité des caractères étudiés. Il est complété par les graphiques de la figure 2 concernant la hauteur et le débourrement de $4^{*}$ année.

A

$5 \%$

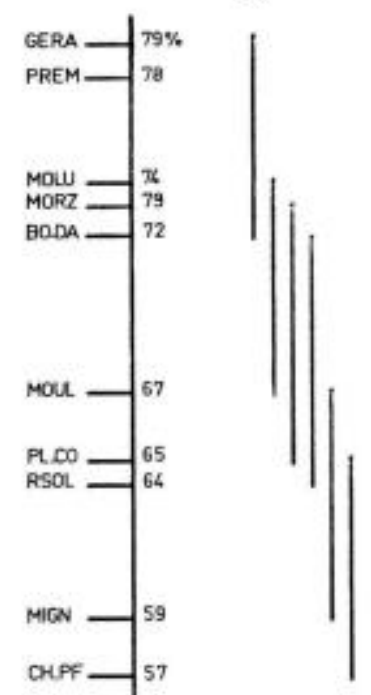

B

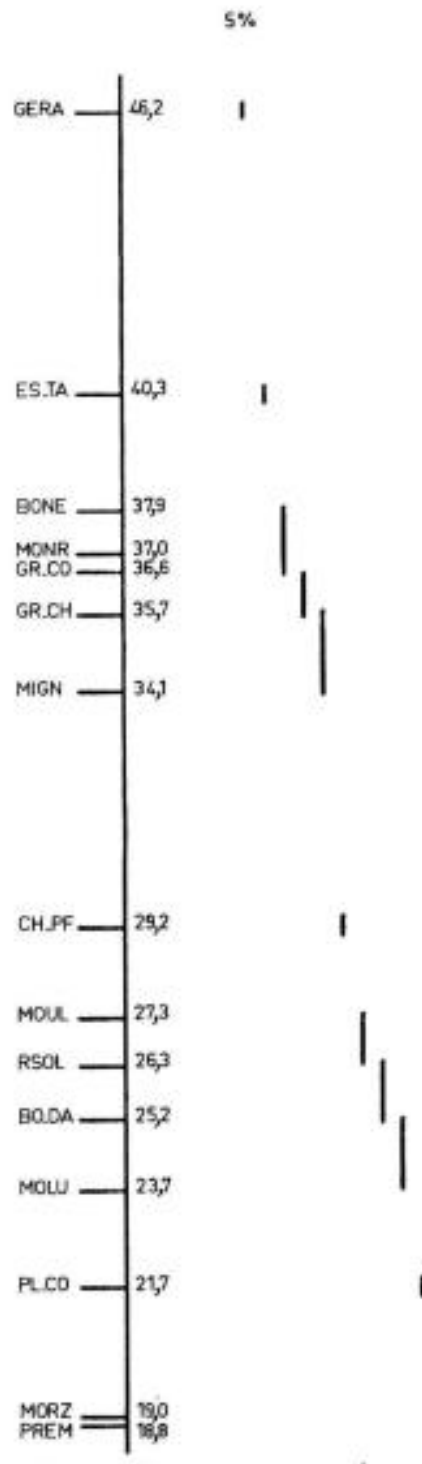

Fig. 2. - Hauteurs $(B)$ et débourrement $(A)$ de $4^{\prime}$ année, au niveau moyennes de provenances. Fic. 2. Tauteurs $(B)$ et débourrement $(A)$ dé $4^{\prime}$ annééles do la $2^{*}$ série de notations.

Fic. 2. - Height $(B)$ and flushing $(A), 4$ th year (means of provenances). 4 th year flushing data are those of the 2 nd notation. 


\subsection{Dimensions et poids secs (vigueur).}

Les hauteurs à 3 et 4 ans, le diamètre et les poids secs (tige et aiguilles) fournissent des classements de provenances très semblables. Les hauteurs à 3 et 4 ans ont un pouvoir de discrimination très élevé (valeurs fortes du $\mathrm{F}$ inter-provenances), le diamètre comme les poids secs (échantillonnage réduit) ont un pouvoir de discrimination plus faible. Ces divers classements traduisent une variabilité d'ordre géographique et altitudinal; par ordre de vigueur décroissante, les provenances se rangent comme suit :

\section{- Vosges (Gérardmer) ;}

- $2^{*}$ plateau du Jura et provenances d'altitude des Préalpes du Nord;

- Hautes chaînes du Jura et provenance de haute altitude des Préalpes du Nor $\mathbf{3}$ (Morzine).

\subsection{Densité du bois de tige '11.}

Au niveau provenance, la densité diminue lorsque la vigueur augmente. Dans les conditions de l'expérience (échantillonnage réduit), c'est un caractère à faible pouvoir de discrimination.

\subsection{Phénologie.}

Débourrement. - La somme des quatre notes de débourrement (au printemps de $4^{*}$ année) et la $2^{*}$ note fournissent des classements pratiquement identiques, par ailleurs très semblables à celui des dégâts de gelées observés à la même époque. On discerne deux groupes:

- $2^{*}$ plateau du Jura (Mignovillard excepté) et provenances d'altitude des Préalpes du Nord, à débourrement tardif, les provenances les plus tardives donc les plus intéressantes étant Montriond (Préalpes du Nord) et Bonnétage (Jura, $2^{*}$ plateau) :

- hautes chaînes du Jura (plus Mignovillard), haute altitude des Préalpes du Nord (Morzine) et Vosges (Gérardmer), à débourrement précoce.

Pousse d'août. - L'aptitude à produire des pousses d'août s'exprime de façon plus ou moins nette suivant les conditions climatiques de l'année d'observation, mais elle demeure génétiquement pré-conditionnée; au niveau de l'échantillonnage de provenances utilisé, cette aptitude augmente avec la vigueur. Le pouvoir de discrimination de ce caractère est ici relativement élevé.

\subsection{Morphologie.}

Couleur des aiguilles. - La fréquence des individus à aiguilles vert-glauque (bleuté) diminue avec la vigueur des provenances. Elle atteint son maximum (36 à $46 \%$ ) chez les provenances des hautes chaines du Jura et Morzine (Préalpes du Nord,

(1) Mesures effectuées par la Station de Recherches sur la Qualité des Bois du C.N.R.F. (méthode de saturation intégrale). 
haute altitude) ; elle est faible ( 14 à $33 \%$ ) chez les provenances du 2 plateau du Jura et les provenances d'altitude des Préalpes du Nord; elle est pratiquement nulle chez Gérardmer (Vosges).

Il s'agit manifestement d'un caractère lié à l'adaptation aux conditions de haute altitude.

Angle d'insertion des aiguilles sur la pousse terminale. - La fréquence des individus à aiguilles appliquées contre la pousse se montre très influencée par la vigueur des provenances. On note néanmoins quelques différences: la fréquence maximum est atteinte chez Montriond (33\%), Gérardmer ne se classant qu'en seconde position $(22 \%)$; par ailleurs, Mignovillard (Jura, $2^{\circ}$ plateau) montre une fréquence faible comparable à celle des provenances des hautes chaînes du Jura. Les moins vigoureuses des provenances de haute altitude atteignent une fréquence pratiquement nulle (Prémanon, Morzine: $1 \%$ ).

Cette analyse de la variabilité inter-provenances concerne une portion importante de l'aire naturelle française déjà étudiée à plusieurs reprises (LACAzE, 1969; BouvArEL. 1961 et 1962) ; les nouveaux résultats confirment fidèlement les anciens, ce qui a motivé la brièveté de cet exposé. Il faut signaler en complément que la provenance Mignovillard, testée pour la première fois dans cette expérience, manifeste un comportement intermédiaire entre les provenances du $2^{*}$ plateau du Jura et celles des hautes chaînes. A priori, on pouvait être tenté de la rattacher au groupe du $2^{*}$ plateau auquel elle appartient géographiquement. En fait, par sa vigueur relativement faible et son débourrement assez précoce, cette provenance ne peut être utilisée que localement ; son emploi dans les reboisements de basse et moyenne altitude est déconseillé.

\section{2. - Variabilité intra-provenance (individuelle)}

\subsection{Expression de la variabilité génétique des arbres mères.}

Si l'hypothèse de panmixie au sein de chaque provenance est vérifiée et si l'on suppose qu'une descendance maternelle donnée est exclusivement composée de demifrères, sa moyenne fournit une estimation de la valeur génétique de l'arbre mère. Ceci n'est vrai qu'à condition de pouvoir négliger les effets maternels (en particulier grosseur des graines), ce qui semble admissible dès 3 ou 4 ans chez l'épicéa. On peut done obtenir une estimation correcte de la variabilité individuelle dans la population d'origine, pour autant que l'échantillonnage des arbres mères soit effectivement représentatif (1).

L'expérience ne permet pas de tester rigoureusement cette dernière hypothèse ; elle permet toutefois d'apprécier assez grossièrement ses chances de validité. Les figures 3 et 4 représentent les moyennes de familles de sept provenances, pour deux caractères

(1) Dans le cas de descendances maternelles exclusivement composées de demi-frères, la variance inter-familles $\left(\sigma^{2}\right)$ estime alors $1 / 4$ de la variance génétique. 
différents : la hauteur à 4 ans et le débourrement (1). Les provenances ont été choisies de façon à couvrir l'ensemble de la gamme de vigueur, de Gérardmer à Prémanon.

3.22. Les imperfections de l'échantillonnage des arbres mères (effectif réduit, utilisation des arbres * plus 3 ).

On peut penser que la distribution réelle des valeurs génétiques individuelles dans chaque population suit une loi normale; dans cette hypothèse, le nombre réduit d'arbres échantillonnés n'affecterait pas dangereusement l'estimation de la moyenne de la population, mais risquerait de sous-estimer l'amplitude réelle de la variabilité individuelle (2).

En pratique, les graphiques des figures 3 et 4 montrent que dans certaines populations la distribution des moyennes de familles est effectivement irrégulière. Concernant la hauteur à 4 ans, ces distributions sont soit irrégulières mais centrées (Montriond), soit nettement dissymétriques (Esserval-Tartre, Prémanon et surtout La Mouille). La dissymétrie semble encore plus fréquente concernant le débourrement (Montriond. Bonnétage, Esserval-Tartre, La Mouille, Prémanon).

Par ailleurs, on peut se demander quelle est l'influence de l'utilisation d'arbres «plus s (3), en tout ou partie, pour représenter certaines populations ?

A Esserval-Tartre, seuls les arbres I S, 2 S et 11 sont choisis au hasard. Pour la hauteur, $1 \mathrm{~S}$ et 11 sont proches de la moyenne de population, $2 \mathrm{~S}$ s'en écarte; pour le débourrement, ces trois arbres sont encore assez voisins de la moyenne. Dans les deux cas, les arbres * plus s sont répartis de part et d'autre des trois arbres choisis au hasard.

A Bonnétage, les arbres 10 et 11 sont choisis au hasard, 11 est le plus vigoureux de la provenance mais reste moyen au débourrement, 10 est de vigueur moyenne mais il est tardif, l'arbre 3 qui est un arbre * plus s est à la fois précoce et de vigueur très inférieure à la moyenne.

A Montriond, l'arbre 1 qui est le seul arbre * plus s de la population est rigoureusement moyen pour la vigueur et le débourrement.

En conclusion, les arbres \& plus * ne montrent aucune supériorité génétique. On en déduit que :

- leur utilisation en tout ou partie pour représenter certaines populations ne diminue pas la portée des résultats obtenus :

- la sélection phénotypique en forêt doit se limiter à un petit nombre de caractères à forte héritabilité en forêt, la sélection pour les caractères à faible héritabilité en forêt (la vigueur en particulier) ne pouvant être envisagée qu'au niveau des plantations comparatives de descendances.

\subsection{Quelques exemples de variabilité génétique individuelle.}

En gardant présente à l'esprit la sous-estimation probable de l'amplitude de la variabilité individuelle au sein de chaque population, on peut néanmoins faire les remarques suivantes concernant les figures 3 et 4 .

(1) Fréquence des individus débourrés lors de la $2^{*}$ série de notations, au printemps de la 4* année de végétation.

(2) En effet la possibilité de tirage est maximum pour les individus voisins de la moyenne.

(3) Voir note, page 143 . 


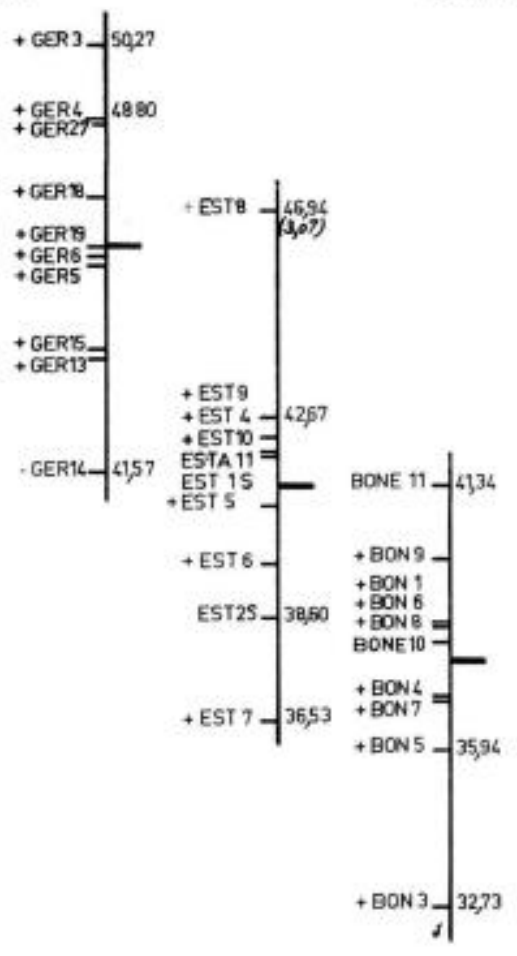

MONR -70

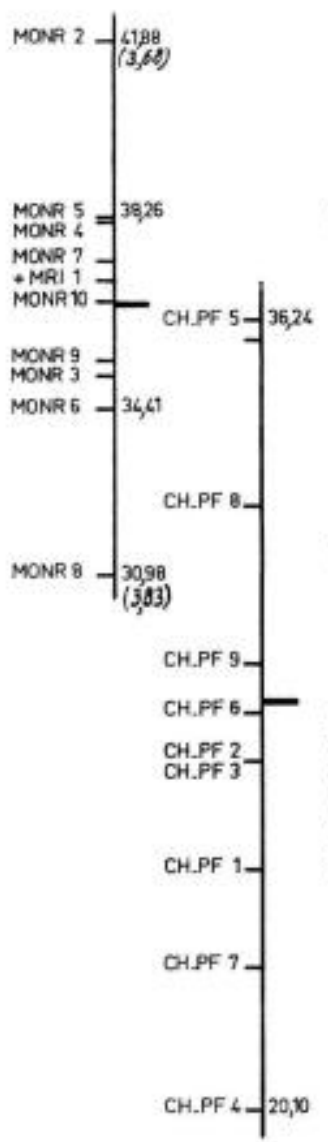

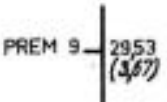

Moul 2

MOUL ?

MOUL 8

MOUL 1 -

MOU $6 \_25,10$

MOUL $6=25,1$

PREM 1-2325

PREM 7

PREM 8 PAEM \&

PAEM

PREM 5

PREM 5

PAEM 3

PFEMD -498

F16. 3. - Quelques exemples de variabilité génétique individuelle: hauteur à 4 ans Pour chaque provenance, le repère en trait fort porté à droite de l'échelle indique la moyenne des 10 descendances

Entre parenthèses, sont indiquées les valeurs de l'écart réduit des descendances extrèmes

FıG. 3, - Some examples of individual genetic variability: 4 years height

For each provenance, the thick line (on the right side of the scale) shows the progenies mean 

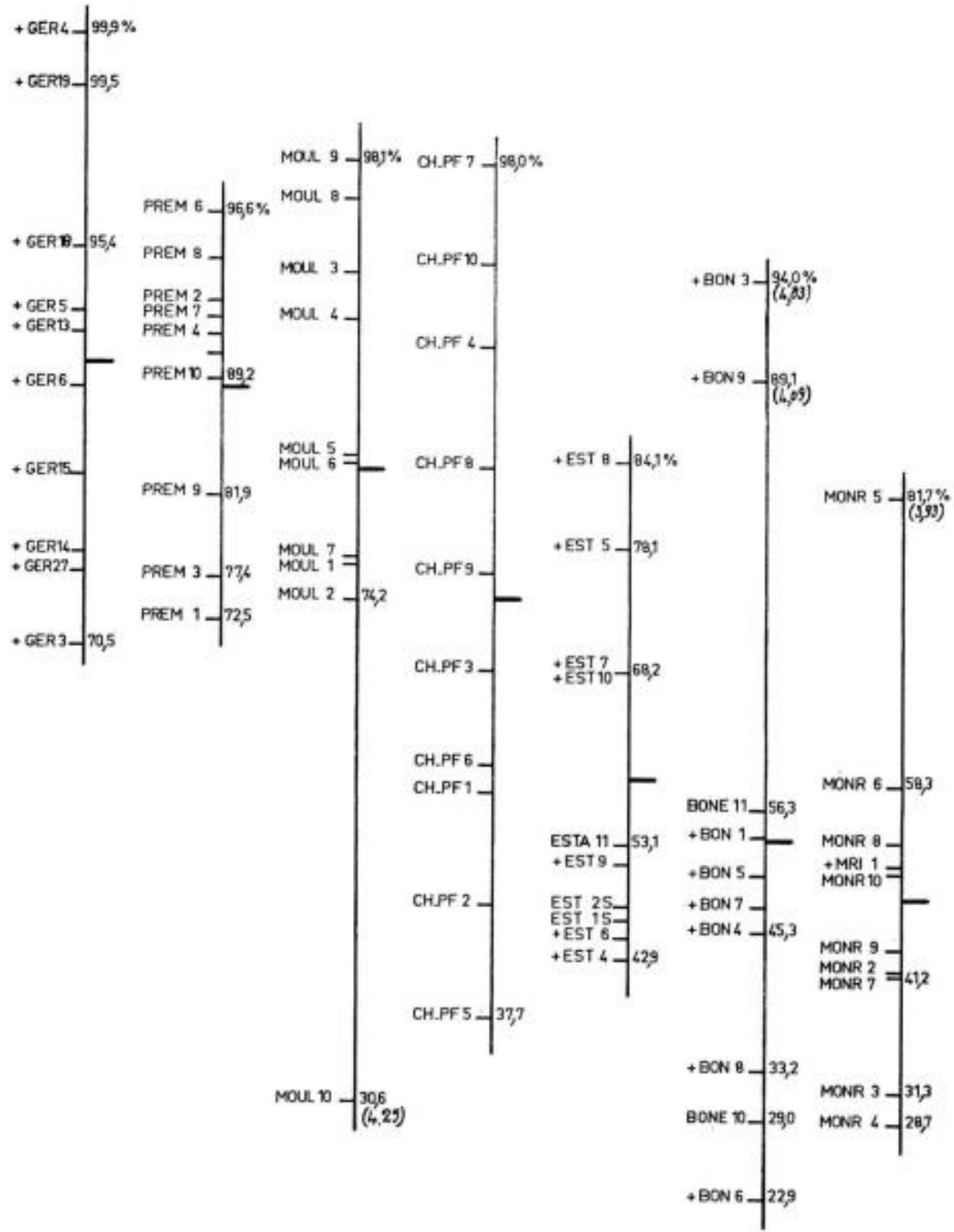

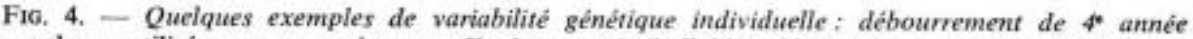
Les valeurs utilisées correspondent au $\%$ de note 1 (individus débourrés) lors de la seconde série de notations

Pour chaque provenance, le repère en trait fort porté à droite de l'échelle indique la moỵenne des 10 descendances

Fig. 4. - Some examples of individual genetic variability : 4th year flushing $\%$ of flushed individual (note 1) during 2nd notation

For each provenance, the thick line (on the right side of the scale) shows the progenies mean 
Moyennes et variances: caractères quantitatifs

Means and variances - Quantitative characters

\begin{tabular}{|c|c|c|c|c|c|c|}
\hline & $\begin{array}{c}\text { 1. Hauteur } \\
3 \text { ans } \\
\text { [Height } \\
\text { at } 3 \text { years] }\end{array}$ & $\begin{array}{c}2 \text { - Hauteur } \\
4 \text { ans } \\
\text { [Height } \\
\text { at } 4 \text { years] }\end{array}$ & $\begin{array}{l}\text { 3- Diamètre } \\
4 \text { ans } \\
\text { [Collar } \\
\text { diameter } \\
\text { at } 4 \text { years] }\end{array}$ & $\begin{array}{c}\text { 4- Poids sec } \\
\text { tige } \\
\text { [Stem dry } \\
\text { weight] }\end{array}$ & $\begin{array}{l}\text { 5- Poids sec } \\
\text { aiguilles } \\
\text { [Needles dry } \\
\text { weight] }\end{array}$ & $\begin{array}{l}\text { 6. Densité } \\
\text { [Wood density] }\end{array}$ \\
\hline $\begin{array}{l}F \text { (provenances) (1) } \ldots \ldots \ldots \ldots \ldots \ldots \ldots \\
F \text { value (provenances) }\end{array}$ & $129,33 * *$ & $148,80 * *$ & 29,64 *e & $43,09=k$ & $33,65 \neq$ & $3,00 * *$ \\
\hline $\begin{array}{l}\text { Nbre DL (trait., err.) ..................... } \\
\text { Degrees of freedom (provenances, error) }\end{array}$ & $14 \quad 987$ & $14 \quad 987$ & $14 \quad 754$ & $14 \quad 1034$ & $14 \quad 1034$ & $14 \quad 1034$ \\
\hline
\end{tabular}

Provenances

\begin{tabular}{|c|c|c|c|c|c|c|c|}
\hline 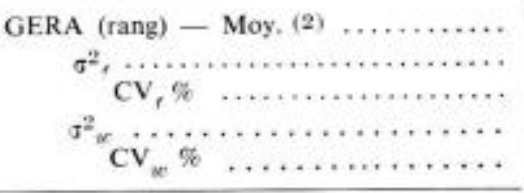 & (1) $\begin{array}{c}20,3 \\
1,5 \\
6,03 \\
53,1 \\
35,9\end{array}$ & $\begin{array}{c}46,5 \\
6,1 \\
5,31 \\
180,9 \\
28,9\end{array}$ & (1) $\begin{array}{l}9,2 \\
0,2 \\
4,81 \\
10,9 \\
35,5\end{array}$ & $\begin{array}{r}12,054 \\
1,374 \\
9,72 \\
17,490 \\
34,7\end{array}$ & (1) $\begin{array}{l}15,474 \\
2,320 \\
9,84 \\
36,971 \\
39,3\end{array}$ & (15) & $\begin{array}{c}519 \\
32 \\
1,08 \\
1085 \\
6,35\end{array}$ \\
\hline 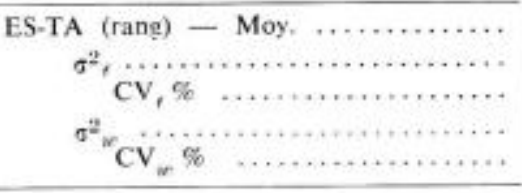 & (2) $\begin{array}{c}17,6 \\
1,1 \\
5,96 \\
31,7 \\
32,0\end{array}$ & (2) $\begin{array}{r}41,3 \\
7,0 \\
6,41 \\
118,3 \\
26,3\end{array}$ & (1) $\begin{array}{c}9,3 \\
0,2 \\
4,81 \\
7,0 \\
28,5\end{array}$ & (2) $\begin{array}{l}9,664 \\
0 \\
0 \\
12,457 \\
36,5\end{array}$ & (2) $\begin{array}{l}13,579 \\
0 \\
0 \\
26,454 \\
37,9\end{array}$ & (13) & $\begin{array}{c}531 \\
\therefore 4 \\
1.25 \\
1.88 \\
\quad 7,26\end{array}$ \\
\hline 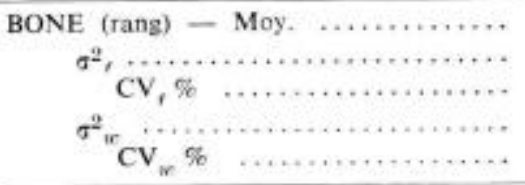 & (4) $\begin{array}{c}15,6 \\
0,7 \\
5,37 \\
29,9 \\
35,1\end{array}$ & (3) $\begin{array}{c}37,7 \\
3,8 \\
5,17 \\
131,9 \\
30,5\end{array}$ & (6) $\begin{array}{l}8,7 \\
0 \\
0 \\
5,8 \\
27,7\end{array}$ & $\begin{array}{l}9,036 \\
1,446 \\
13,3 \\
10,756 \\
363\end{array}$ & (5) $\begin{array}{l}12,201 \\
2,032 \\
11,7 \\
24,508 \\
40.6\end{array}$ & (10) & $\begin{array}{c}536 \\
151 \\
2,29 \\
1587 \\
\quad 7.43\end{array}$ \\
\hline
\end{tabular}




\begin{tabular}{|c|c|c|c|c|c|c|}
\hline 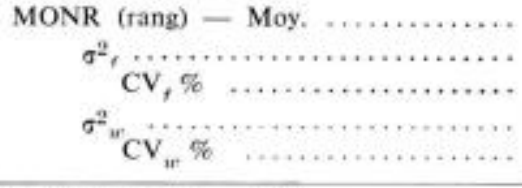 & (3) $\begin{array}{c}15,7 \\
1,3 \\
7,26 \\
31,0 \\
35,5\end{array}$ & (5) $\begin{array}{c}36,5 \\
6,9 \\
7,20 \\
112,2 \\
29,0\end{array}$ & (4) $\begin{array}{c}8,9 \\
0,2 \\
5,02 \\
6,2 \\
28,0\end{array}$ & $\begin{array}{l}9,278 \\
0,128 \\
3,86 \\
16,035 \\
43,1\end{array}$ & (3) $\begin{array}{l}13,288 \\
0 \\
0 \\
41,144 \\
48,2\end{array}$ & $\begin{array}{r}533 \\
185 \\
2,55 \\
1104 \\
6,23\end{array}$ \\
\hline 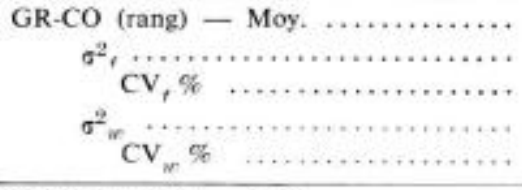 & (5) $\begin{array}{c}15,2 \\
2,0 \\
9,30 \\
33,3 \\
38,0\end{array}$ & (4) $\begin{array}{c}36,7 \\
9,2 \\
8,26 \\
122,5 \\
30,2\end{array}$ & (3) $\begin{array}{c}9,1 \\
0,1 \\
3,47 \\
7,6 \\
30,3\end{array}$ & (5) $\begin{array}{c}8,860 \\
0,154 \\
4,42 \\
8,547 \\
33,0\end{array}$ & (4) $\begin{array}{l}13,041 \\
0 \\
0 \\
20,027 \\
34,4\end{array}$ & $\begin{array}{c}528 \\
52 \\
1,37 \\
1424 \\
7,15\end{array}$ \\
\hline 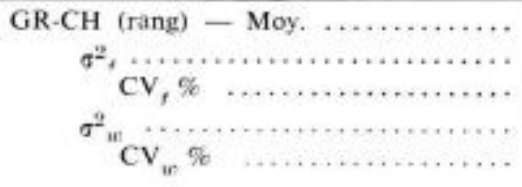 & $\begin{array}{r}14,6 \\
3,9 \\
13,5 \\
26,3 \\
35,1\end{array}$ & (6) $\begin{array}{r}35,0 \\
25,2 \\
14,3 \\
115,7 \\
30,7\end{array}$ & (5) $\begin{array}{c}8,8 \\
0,4 \\
7,19 \\
6,8 \\
29,7\end{array}$ & (6) $\begin{array}{l}7,999 \\
0,728 \\
10,7 \\
10,271 \\
40,0\end{array}$ & (6) $\begin{array}{l}11,900 \\
1,091 \\
8,78 \\
26,554 \\
43,3\end{array}$ & $\begin{array}{c}537 \\
104 \\
1,89 \\
1071 \\
6,09\end{array}$ \\
\hline
\end{tabular}

(1) Résultats tirés des analyses de variance en blocs incomplets sur les moyennes de sous-parcelles (il $y$ avait en principe $15 \times 10 \times 7=1050$ sous-parcelles, mais en fait quelques données manquantes: l'analyse de variance a donc été effectuée suivant un modèle non orthogonal).

Comme les analyses portaient sur des moyennes, les caractères qualitatifs suivants (tableau 3 B) ont été étudiés sous forme de pourcentages après transformation en are sin $V:$ Débourrement $(\%$ note 1$)$, Dégâts de gel (\% note 2$)$, Pousse d'août de $3^{*}$ et $4^{*}$ année (\% notes $\left.2+3\right)$, Couleur (\% note 1 ). Angle d'insertion des aiguilles ( $\%$ note 2 ).

(2) Résultats tírés des analyses de variance effectuées sur les données individuelles suivant un modèle à un facteur contrôlé (cf. §̧ 61 du texte). $\sigma^{2},=$ variance entre familles; $\mathrm{CV}_{t}=$ coefficient de variation entra familles

$\sigma^{2}{ }_{w}=$ variance résiduelle; $\mathrm{CV}_{w}=$ coefficient de variation résiduel.

Comme il s'agit de données individuelles, les caractères qualitatifs (tableau 3 B) ont été utilisés directement sous forme de notation, sans transformation; en ce qui concerne les caractères 7 et 12, les moyennes sont exprimées en pourcentages.

(1) Results from incomplete blocs analysis of variance (data : means of sub-plot).

(2) Results from one way-analysis of variance (individual data).

(Rank) mean.

$\sigma^{2}$, : between family variance.

$\mathrm{CV}_{i}$ : between family coefficient of variation.

$\sigma^{2} y$ : residual variance

$\mathrm{CV}_{\text {ur }}{ }^{*}$ : residual coefficient of variation. 
TABLEAU 3 A (suite) - TABLE 3 A (continued)

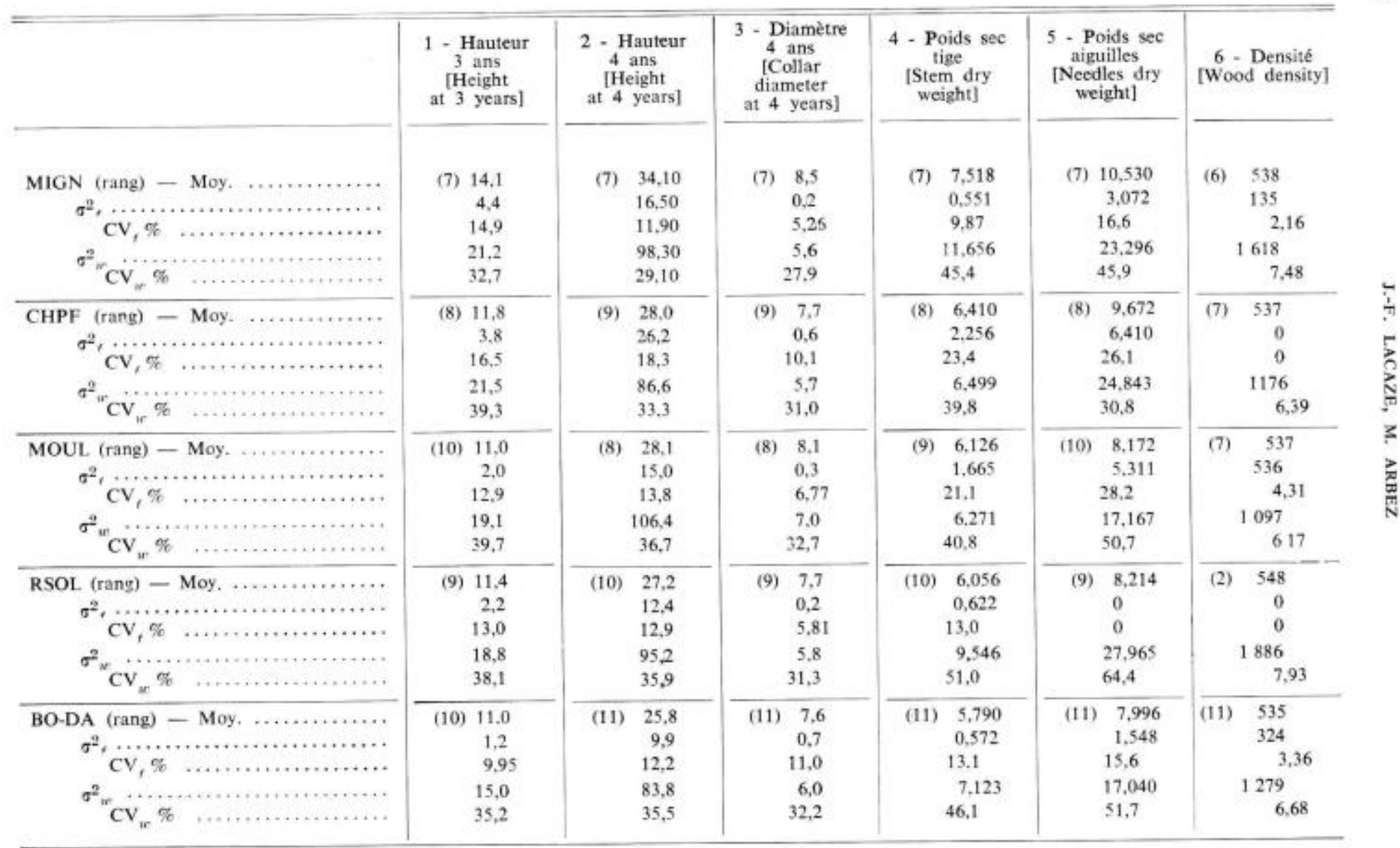




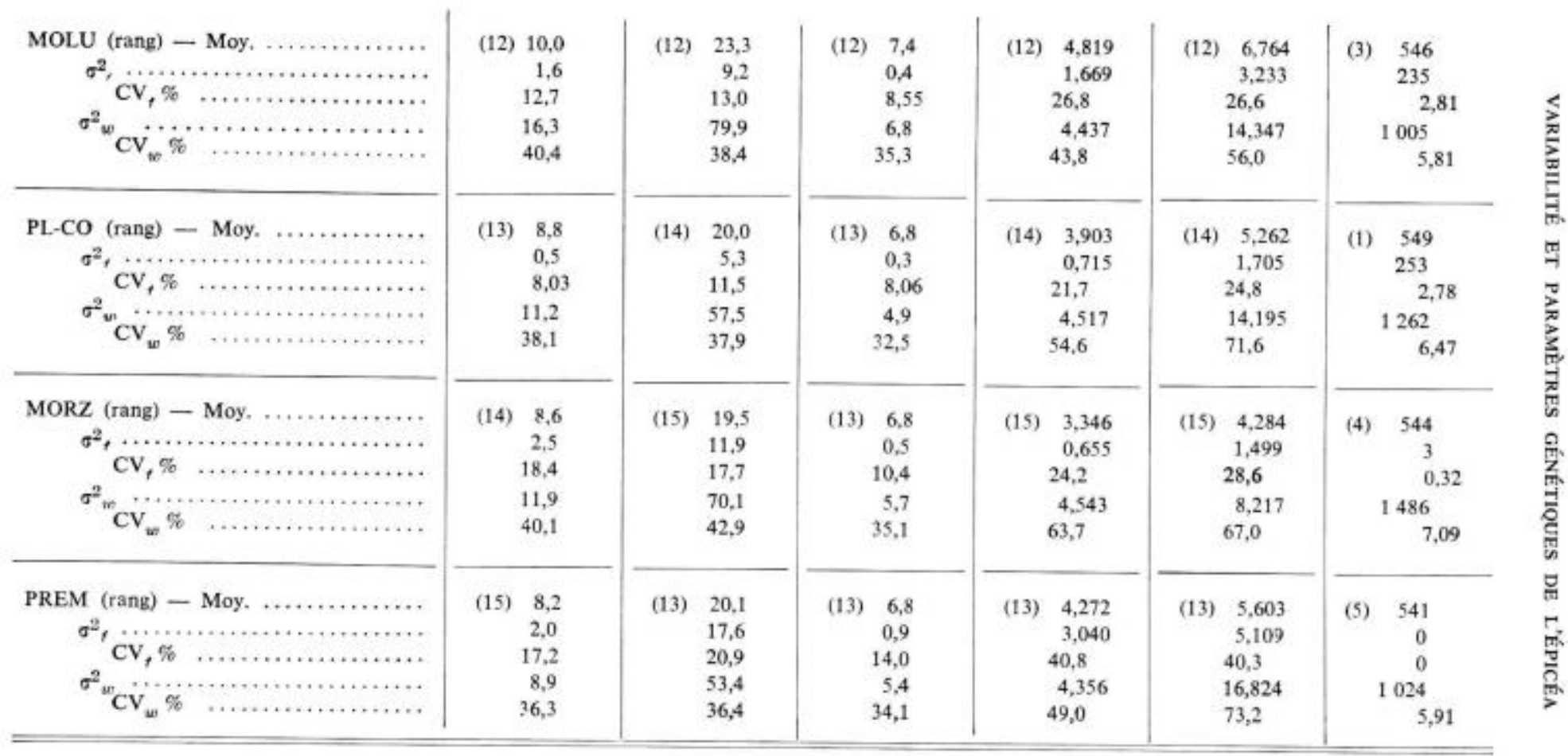


Movennes et variances: caractères qualitatifs

Means and variances - Qualitative characters

\begin{tabular}{|c|c|c|c|c|c|c|c|}
\hline & $\begin{array}{l}7 \text { - Débour- } \\
\text { rement } \\
\text { [Flushing] }\end{array}$ & $\begin{array}{l}8 \text { - Somme } \\
\text { Déb. } \\
\text { [Sum of } \\
\text { flushing } \\
\text { notations] }\end{array}$ & $\begin{array}{l}9 \text { - Dégâts } \\
\text { gel } \\
\text { [Spring } \\
\text { frost } \\
\text { damages] }\end{array}$ & $\begin{array}{l}10 \text { - Pousse } \\
\text { d'août } \\
3^{*} \text { année } \\
\text { [Lamma } \\
\text { shoot } \\
\text { on the } \\
\text { 3rd year] }\end{array}$ & $\begin{array}{l}11 \text { - Pousse } \\
\text { d'aoút } \\
4^{\prime} \text { année } \\
\text { [Lamma } \\
\text { shoot } \\
\text { on the } \\
\text { 4th year] }\end{array}$ & $\begin{array}{c}12 \text { - Couleur } \\
\text { [Needle } \\
\text { color] }\end{array}$ & $\begin{array}{l}13 \text { - Angle } \\
\text { d'insertion } \\
\text { [Angle of } \\
\text { insertion } \\
\text { of needles] }\end{array}$ \\
\hline 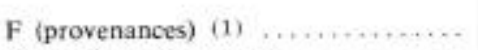 & $25,23 * *$ & & $15,94 * *$ & $27,05 * *$ & $20,91 * *$ & $35,35 * \Rightarrow$ & $24,25 * *$ \\
\hline Nbre DL (trait., err.) ,........... & $14 \quad 1001$ & & $14 \quad 1001$ & $14 \quad 987$ & $14 \quad 1001$ & $14 \quad 1001$ & $14 \quad 987$ \\
\hline
\end{tabular}

Provenances

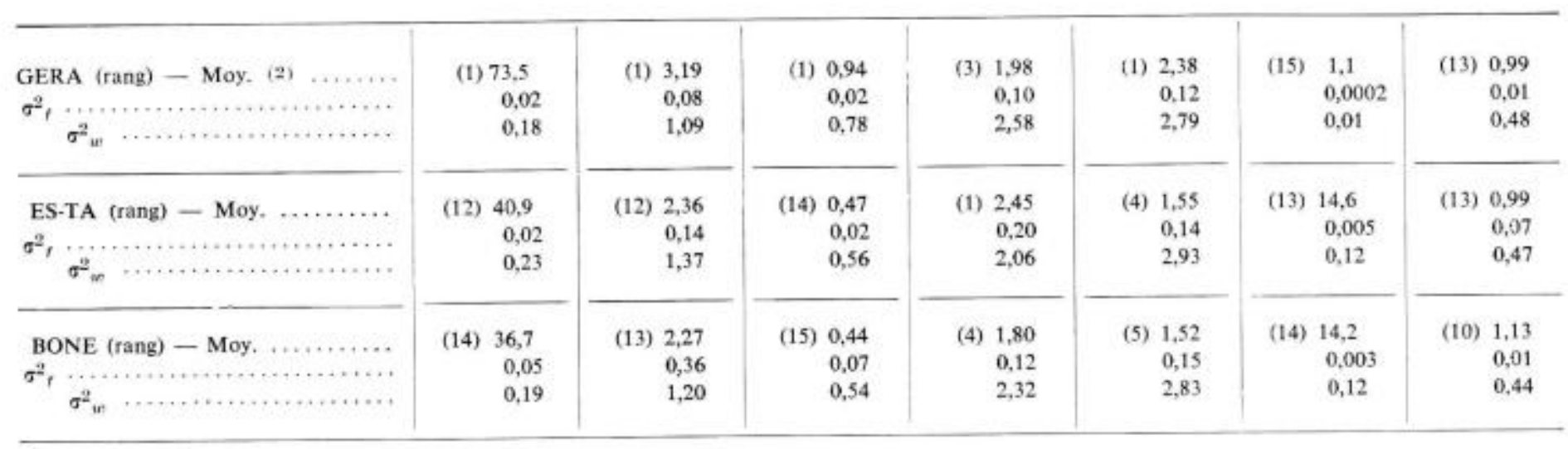




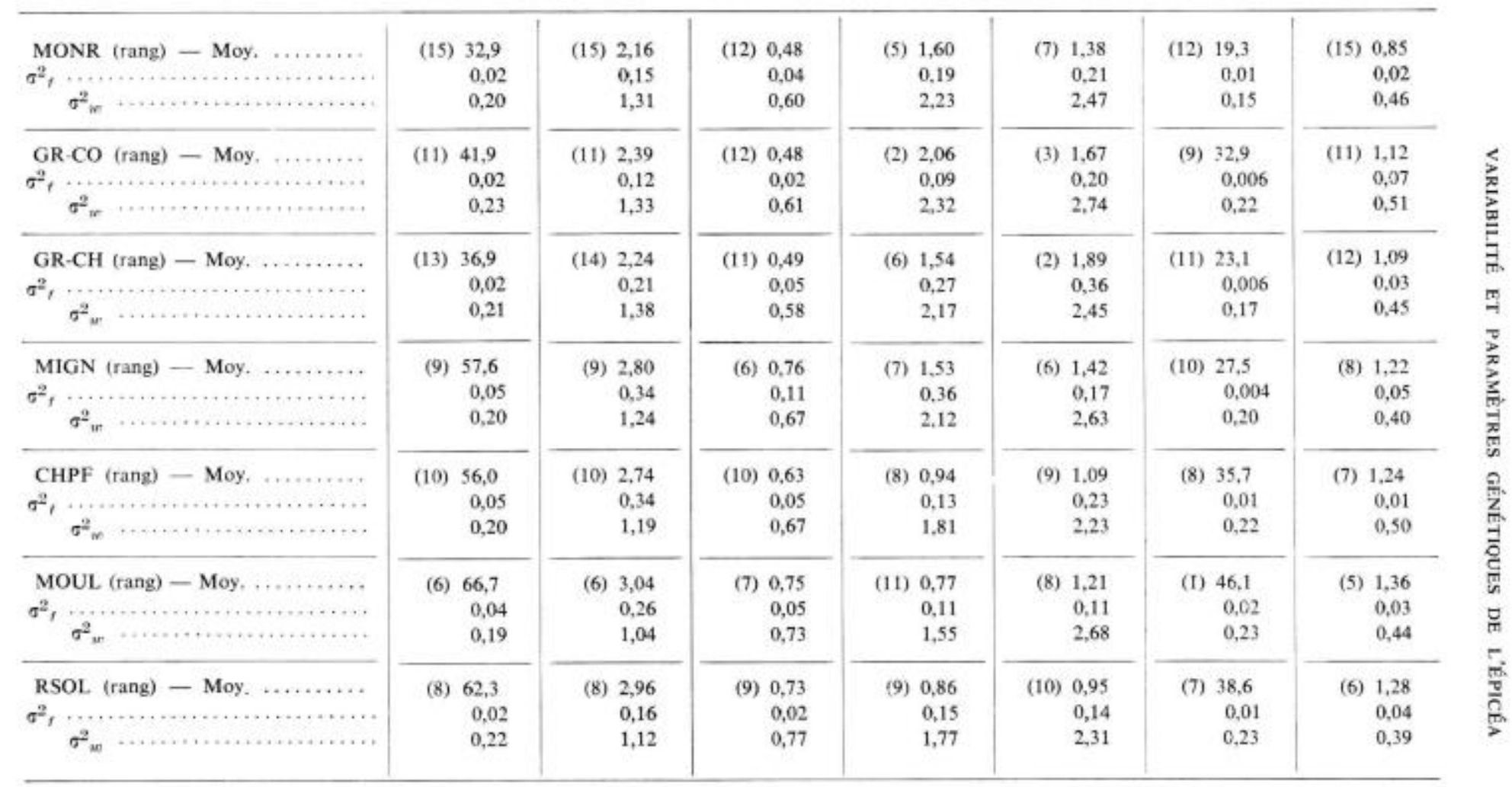

(1) cf. notes du tableau $3 \mathrm{~A}$. 
Tableau 3 B (suite) Table 3 B (continued)

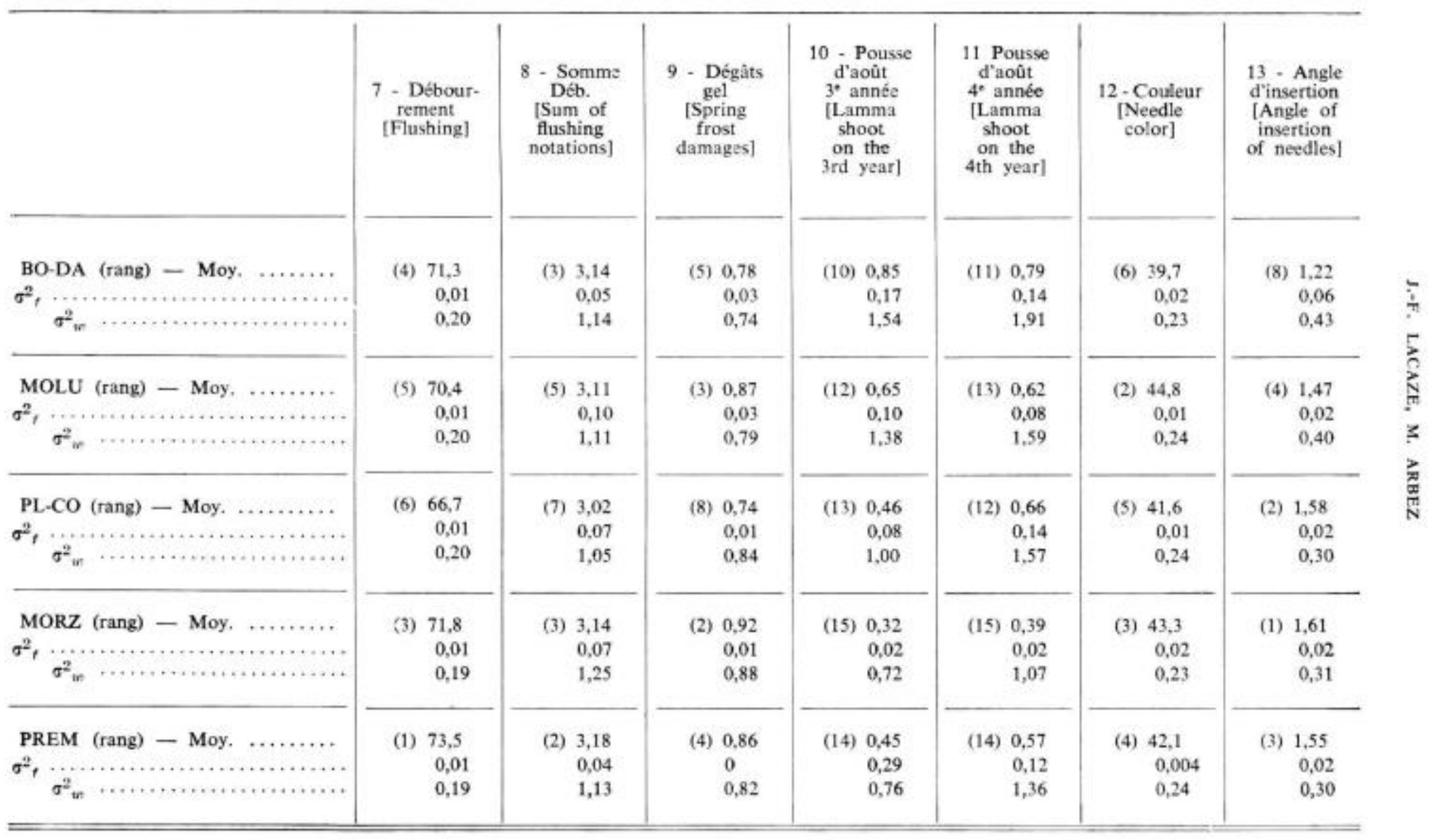


Hauteur à 4 ans (figure 3). - Dans l'exemple choisi, l'amplitude des écarts entre familles d'une même provenance varie de 10 à $15 \mathrm{~cm}$; elle est donc comprise entre ic tiers et la moitié de l'amplitude de variabilité totale, inter-populations $(27 \mathrm{~cm})$.

Cette variabilité inter-familles, qui exprime la variabilité génétique individuelle en forêt, est beaucoup plus élevée dans les populations peu vigoureuses de haute altitude.

Les moyennes de familles de deux provenances voisines au classement se chevauchent presque toujours. Dans certains cas particuliers, la meilleure famille de la population la moins vigoureuse peut même surclasser son homologue de la population la plus vigoureuse (voir $\mathrm{La}$ Mouille et Champfromier).

Débourrement (figure 4). - L'amplitude des écarts entre familles d'une même provenance est encore plus considérable pour ce dernier caractère. Dans l'exemple choisi, elle varie entre $24 \%$ (Prémanon) et $68 \%$ (La Mouille) contre $48 \%$ seulement entre les moyennes des deux provenances extrêmes (Gérardmer et Montriond).

Dans ces conditions, la meilleure famille de Gérardmer est plus tardive que ia moins bonne de Montriond.

\subsection{Comparaison des variances inter-familles dans chaque provenance.}

Les estimations des variances inter-familles dans chaque provenance $\left(\sigma^{2} f\right)$, obtenues par analyse et décomposition de la variance (modèle à un facteur contrôlé : descendances dans provenance, cf. $\S 61$ ), figurent au tableau 3. Les coefficients de variation correspondants ont été calculés pour les seules variables quantitatives (vigueur et densité). Ces résultats suscitent les remarques suivantes:

- Concernant les caractères de vigueur, les coefficients de variation entre familles sont notablement plus élevés chez les provenances peu vigoureuses de haute altitude (1).

- Cette opposition systématique entre populations de vigueurs différentes disparaît lorsqu'on examine les valeurs des variances inter-familles des caractères phénologiques ou morphologiques. Concernant les caractères phénologiques (débourrement et pousse d'août), Bonnétage et Mignovillard montrent une variabilité inter-familles très élevée (traduisant une variabilité génétique entre individus, dans les populations d'origine, elle-même très élevée).

\section{3. - Variabilité résiduelle}

\subsection{Interprétation.}

Elle correspond à la variance intra-famille (observée entre individus d'une même famille).

Elle regroupe des effets de nature très différente (effets génétiques (2), effets du milieu et de concurrence, erreurs de mesures).

Son interprétation est donc délicate et la part de variabilité qu'elle absorbe est inutilisable dans la plupart des schémas d'amélioration par voie sexuée.

(1) La provenance Grande Chartreuse, bien qu'assez vigourcuse, se singularise néanmoins par des valeurs importantes du coefficient de variation inter-familles.

(2) Dans le cas de familles maternelles constituées exclusivement de demi-frères, la part génétique de la variance résiduelle représente les $3 / 4$ de la variance additive et la plus grande partie de la variance non additive. 
Les valeurs obtenues par analyse et décomposition de la variance (modèle à un facteur contrôlé : descendances dans provenance, cf. \& 61 ) sont consignées au tableau 3 .

\subsection{Différences entre provenances.}

On compare les variances résiduelles moyennes de chaque provenance (moyenne des variances résiduelles des 10 familles qui la composent).

Caractères quantitatifs (densité exceptée), - Les résultats du tableau 3 montrent que, pour un caractère quantitatif donné, la variance résiduelle augmente avec la grandeur de la moyenne. On désigne habituellement ce phénomène sous le nom d'effet d'échelle (FALCONER, 1960). Le coefficient de variation correspondant varie peu et l'on pourrait considérer cette relation comme linéaire, en première approximation.

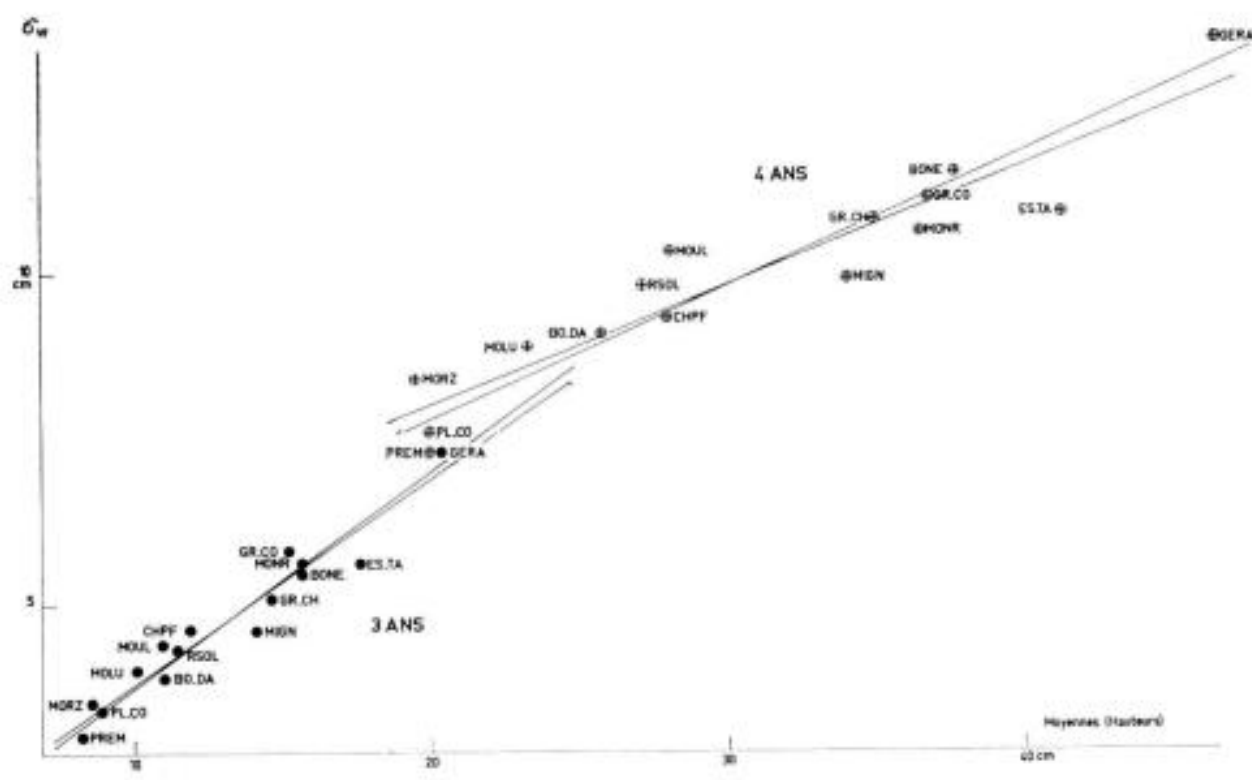

Fic. 5. - Variation de Pécart-rype résiduel en fonction de la moyenne (hauteurs à 3 et 4 ans) FIG. 5, - Variation of residual standard deviation with the mean of height ( 3 and 4 years)

En examinant les choses de plus près, on constate qu'excepté dans le cas du diamètre, le coefficient de variation résiduel diminue lorsque la moyenne augmente. L'écarttype résiduel augmente done proportionnellement moins vite que la moyenne, ce qui tendrait à prouver soit que la relation est en fait curvilinéaire, soit que les provenances de haute altitude d'une part, de moyenne altitude d'autre part, suivent des lois différentes. La figure 5 concerne, à titre d'exemple, les hauteurs à 3 et 4 ans.

Dans le cas du diamètre, la loi de variation de l'écart-type résiduel en fonction de la moyenne (fig. 6) est plus complexe. L'hypothèse de deux groupes distincts suivant des lois de variation approximativement linéaires, mais différentes, s'impose avec plus de force que précédemment. De plus, la provenance Gérardmer semble se séparer du groupe de moyenne altitude par un écart-type résiduel anormalement élevé. Il est 
tentant d'attribuer à la concurrence entre individus l'explication de ce comportement singulier. En effet, on peut penser que l'intervention de la concurrence, d'autant plus probable que la provenance est plus vigoureuse, se traduit par une augmentation supplémentaire de l'écart-type résiduel.

En résumé, une part importante (1) des différences de variances résiduelles observées entre provenances est expliquée par un simple effet d'échelle. Dans ces conditions, on peut penser qu’à hauteur égale (même stade de développement), les variances résiduelles seraient assez peu différentes d'une provenance à lautre. C'est ce que l'on vérifie effectivement dans un cas particulier: la hauteur à 3 ans de Gérardmer et la hauteur à 4 ans de Prémanon et Plan des Cosaques, sont pratiquement égales et les variances résiduelles correspondantes le sont aussi.

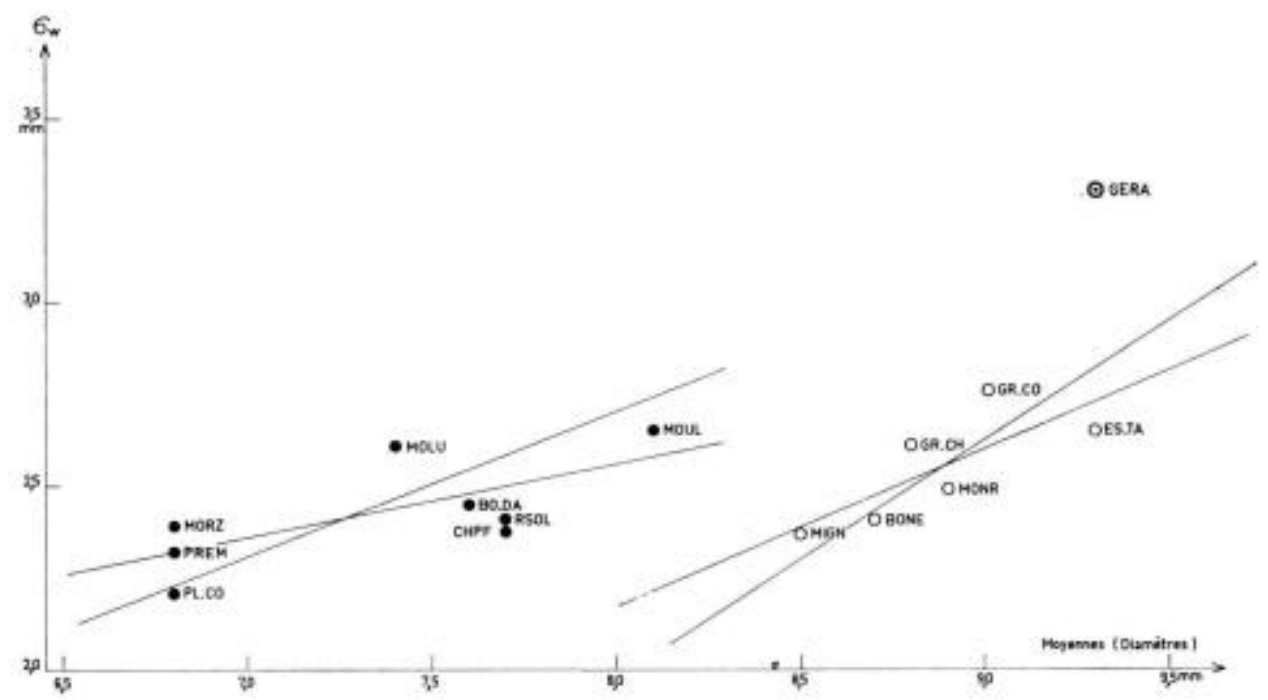

FIG. 6. - Variation de Pécart-type résiduel en fonction de la moyenne (diamètre au collet à 4 ans) Fio. 6. - Variation of residual standard deviation with the mean of collar diameter (4 years)

Il y a tout lieu de croire que l'effet d'échelle affecte aussi les variances entre familles $\left(\sigma^{2} f\right)$. Toutefois, pour un même caractère à âge égal, le phénomène est artificiellement masqué par les grandes différences d'ordre génétique qui existent effectivement entre provenances concernant lamplitude de la variabilité entre familles. On vérifie cependant son existence en constatant que le coefficient de variation entre familles dans la même provenance reste pratiquement constant entre 3 et 4 ans pour les hauteurs. L'effet d'échelle affectant à la fois $\sigma_{f}^{2}$ et $\sigma^{2}{ }_{w}$, les estimations d'héritabilité ne devraient pas en être modifiées. Cette remarque autorise donc à comparer les valeurs d'héritabilité obtenues à âge égal mais pour des populations de hauteur différente.

(1) Hauteurs à 3 ans: $94 \%(r=0,97)$,

ì 4 ans: $88 \%(r=0,94)$.

Diamétre à 4 ans: $65 \%$ provenances de moyenne altitude $(r=0,81)$.

$50 \%$ provenances de haute altitude $(r=0,71)$. 
Caractères qualitatifs et densité. - Dans le cas de la densité, caractère quantitatif d'un type particulier puisqu'il s'agit en fait d'un rapport, on ne trouve aucune relation entre la variance résiduelle et la moyenne.

Dans le cas des caractères qualitatifs, il n'existe aucune règle générale. Toutes les possibilités sont effectivement représentées; s'agissant de notation, le fait n'est pas étonnant :

- indépendance entre la variance et la moyenne ( $2^{\circ}$ notation de débourrement) ;

- liaison positive (dégât de gel, pousse d'août de $3^{\circ}$ et $4^{\circ}$ année, couleur) ; aiguilles).

- liaison négative (somme des notes de débourrement, angle d'insertion des

Lorsqu'il existe une liaison, elle est généralement assez faible.

\subsection{Différences entre familles de la même provenance.}

Nous nous sommes limités à deux caractères: hauteur et somme des notations de débourrement à 4 ans.

Concernant le débourrement, dans la majorité des provenances il y a indépendance entre la variance résiduelle d’une descendance et sa moyenne.

Concernant la hauteur, la liaison entre ces deux paramètres est suivant les cas nulle ou faible (la variance conservant une certaine tendance à augmenter avec la valeur de la moyenne). Quand il subsiste, l'effet d'échelle n'explique plus qu'une part très réduite des différences de variance résiduelle observées entre descendances. Or, dans une même provenance, les différences de coefficient de variation résiduel s'échelonnent entre $7 \%$ (Gérardmer, Bonnétage, Champfromier) et $20 \%$ (Grand-Côte, La Mouille, Les Molunes).

Compte tenu de la relative homogénéité des conditions de milieu en pépinière et des répétitions du dispositif, on peut penser qu'effectivement certaines familles sont génétiquement plus variables que d'autres. Le nombre et le degré de parenté des pères qui sont effectivement intervenus dans la pollinisation de l'arbre mère peuvent en partie contribuer à expliquer ces différences.

\section{4. - RÉPARTITION DE LA VARIABILITÉ ENTRE LES DIFFÉRENTS NIVEAUX}

\section{1. - Méthode de calcul}

Les calculs ont été effectués par la Station de Biométrie du C.N.R.F., sur ordinateur IMB 1130, en utilisant le programme HIERA qui effectue l'analyse et la décomposition des variances selon le schéma hiérarchique suivant (1) :

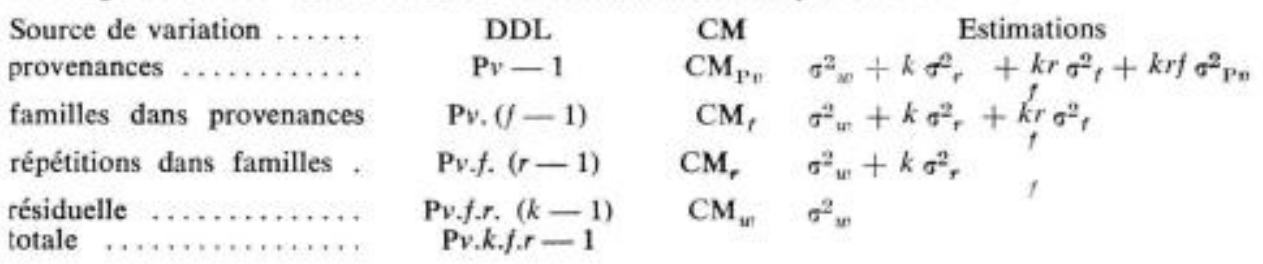

(1) $\mathrm{Ce}$ programme effectue par ailleurs, de façon semblable, l'analyse et la décomposition des covariances. Il a été mis au point par la Station de Génétique animale de II.N.R.A. à Jouy-enJosas. 
avec DDL nombre de degrés de liberté,

CM carré moyen,

Pv nombre de provenances (15).

$f$ nombre de familles dans chaque provenance (10),

$r \quad$ nombre de répétitions (7),

$k$ nombre d'individus par parcelle unitaire (16),

$\sigma^{2} i^{2} \quad$ variance entre provenances,

$\sigma^{2}$, variance entre familles dans chaque provenance,

$\sigma^{2}$ r variance entre répétitions dans chaque famille,

$\sigma^{2} \quad$ variance résiduelle.

Ce schéma est critiquable dans la mesure où l'effet répétition dans famille est considérć comme aléatoire alors qu'il s'agit en réalité d'un effet fixe.

\section{2. - Résultats}

Nous limiterons l'exposé des résultat; à deux caractères seulement: la hauteur à 4 ans et le débourrement.

\section{TABLEAU 4 - TABLE 4}

Répartition de la variabilité entre les différents niveaux Distribution of the variability between the different levels

\begin{tabular}{|c|c|c|}
\hline$\underset{\text { (Characters) }}{\text { Caractères }}$ & $\begin{array}{l}\text { Hauteur aे } 4 \text { ans } \\
\text { (Height at } 4 \text { years) }\end{array}$ & $\begin{array}{l}\text { Débourrement * } \\
\text { (Flushing) }\end{array}$ \\
\hline $\begin{array}{l}\text { Var. entre prov. } \sigma_{\mathrm{p}^{y}}^{2} \ldots \ldots \ldots \ldots \ldots \ldots \ldots \\
\text { Between prov.-Var. }\end{array}$ & $62.00(35,7 \%)^{* *}$ & $0,1241(8,4 \%)$ \\
\hline $\begin{array}{l}\text { Var, entre familles dans prov. } \sigma_{1}^{3} \ldots \\
\text { Between families within prov,-Var. }\end{array}$ & $9.64(5,6 \%)$ & $0,1593(10,7 \%)$ \\
\hline $\begin{array}{l}\text { Var, entre répétitions dans famille } \sigma_{r / t}^{2} \\
\text { Between replications within family-Var. }\end{array}$ & $17.90(10,3 \%)$ & $0.0501(3,4 \%)$ \\
\hline $\begin{array}{l}\text { Var. résiduelle } \sigma_{\mathrm{w}}^{2} \ldots \ldots \ldots \ldots \ldots \ldots \\
\text { Residual var. }\end{array}$ & $83.91(48,4 \%)$ & $1,1503(77,5 \%)$ \\
\hline $\begin{array}{l}\text { Var, totale } \ldots \ldots \ldots \ldots \ldots \ldots \ldots \ldots \\
\text { Total var. }\end{array}$ & 173.45 & 1,4838 \\
\hline
\end{tabular}

- Somme des quatre notes de débourrement.

Sum of the 4 notes of flushing. niveaux.

*4 Les chiffres entre parenthèses indiquent le pourcentage de la variance totale aux différents

Figures within brackets mean the percentage of total variance corresponding with the different levels of variations.

Il convient de ne pas oublier que ces résultats s inscrivent dans un contexte expérimental donné et ne peuvent pas être généralisés. Néanmoins, pour l'épicéa dans la portion française de son aire naturelle, ils représentent les seules informations disponibles sur la répartition de la variabilité aux différents niveaux. 


\section{Pour la hauteur à 4 ans.}

La variance résiduelle, donc incontrôlable, représente près de la moitié de la variance totale. La variabilité due aux effets contrôlés de l'environnement (répétition) n'est pas négligeable $(10 \%)$ surtout compte tenu de l'homogénéité du milieu en pépinière.

La part de variabilité due aux différences entre provenances est importante $(35 \%)$; elle est intégralement fixée et directement utilisable. Au moins pour la vigueur, la sélection des provenances fournirait en première étape le gain génétique maximum. La sélection individuelle apporterait un gain supplémentaire relativement modeste, parce que la variabilité génétique individuelle est faible comparée à celle qui existe entre populations et parce qu'elle n'est que partiellement utilisable (valeur réduite de l'héritabilité en forêt).

\section{Pour le débourrement.}

La part de la variance résiduelle est énorme $(78 \%)$; elle est probablement surestiméc par la méthode utilisée pour appréhender le phénomène. Le débourrement est très peu influencé par les variations contrôlées du milieu en pépinière $(3 \%)$. Contrairement à ce qui se passe pour la vigueur, la variance entre provenances $(8 \%)$ est plus faible que la variance entre familles dans les provenances $(11 \%)^{(1)}$. La sélection de provenances en première étape se justifie toujours ${ }^{(2)}$, mais le gain supplémentaire dû à la sélection individuelle sera plus fort que pour la vigueur, pour autant que l'héritabilité correspondante soit bonne.

\section{5. - ClasSIFICATION DES POPUlations}

On a effectué une analyse des composantes principales sur les moyennes de provenances de 9 caractères $\left(\mathrm{n}^{* *} 1,2,7,8,9,10,11,12,13\right)$. Un premier essai ayant révélé la grande originalité de la provenance Gérardmer, on a procédé à un deuxième essai sans en tenir compte, soit avec 14 provenances seulement. Cette analyse a donné les résultats suivants (tableau 5):

- La première composante représente $90,5 \%$ de la variation totale; elle explique donc à elle seule l'essentiel de la variabilité. Elle est également répartie sur les 9 caractères (avec une très légère prépondérance des deux hauteurs).

- La deuxième composante n'est pas significativement différente de zéro; elle ne représente en effet que $3,4 \%$ de la variation totale. Elle est encore assez bien répartie sur l'ensemble des 9 caractères, mais cette fois avec un poids sensiblement plus fort pour les deux expressions du débourrement.

Les projections des provenances sur le plan des deux vecteurs propres font l'objet de la figure 7. Au cas particulier, on aurait tout aussi bien pu se contenter d'une repré-

(1) Cette variance correspond à celle des arbres-mères dans le peuplement d'origine.

(2) La variance entre provenances est évidemment beaucoup plus importante si l'on considère l'ensemble de l'aire européenñ ; il existe notamment, en Pologne, des provenances à débourrement très tardif dont la sélection conduit à des gains génétiques importanls et immédiats. 
sentation linéaire sur le premier vecteur, statistiquement seul significatif. Les provenances $s^{+} y$ classent, selon leur vigueur, dans un ordre d'ailleurs rigoureusement identique à celui des hauteurs à 4 ans (cf. figure 2). L'utilisation du second vecteur amène un clivage des provenances vigoureuses et intermédiaires (Champfromier et Mignovillard) en fonction surtout de leur comportement au débourrement ; chez les provenances peu vigoureuses de haute altitude, l'apport du second vecteur n'est pas aussi simple à interpréter.

\section{TABLEAU 5 - TABLE 5}

Analyse des composantes principales

(14 provenances, Gérandmer exceptée : 9 caractères)

Principal components analys's

\begin{tabular}{|c|c|c|}
\hline & \multicolumn{2}{|c|}{ Composantes principales } \\
\hline & $Y_{1}$ & $\mathrm{Y}_{2}$ \\
\hline Valeurs propres ........... & 8,152 & 0,305 \\
\hline \% variation ................ & 90,58 & 3,39 \\
\hline \% cumulé .................. & 90,58 & 93,97 \\
\hline \multicolumn{3}{|l|}{$\mathrm{N}^{*}$ des caractères } \\
\hline $1, \ldots \ldots \ldots \ldots \ldots \ldots, \ldots$ & 0,345 & 0,263 \\
\hline $2 \quad \ldots \ldots \ldots \ldots \ldots \ldots \ldots, \ldots \ldots \ldots$ & 0,342 & 0,360 \\
\hline $7 \ldots \ldots \ldots \ldots \ldots$ & $-0,339$ & 0,416 \\
\hline $8 \ldots \ldots \ldots \ldots \ldots \ldots \ldots$ & -0.338 & 0,438 \\
\hline $9 \quad \ldots \ldots \ldots \ldots \ldots \ldots$ & $-0,333$ & 0,357 \\
\hline $10 \ldots \ldots \ldots \ldots \ldots \ldots \ldots$ & 0,333 & 0,390 \\
\hline $11+\ldots \ldots \ldots \ldots \ldots \ldots \ldots \ldots$ & 0,324 & 0,218 \\
\hline $12 \ldots \ldots \ldots \ldots \ldots \ldots \ldots \ldots$ & $-0,322$ & 0,244 \\
\hline $13 \ldots \ldots \ldots \ldots \ldots \ldots \ldots \ldots \ldots$ & $-0,323$ & $-0,223$ \\
\hline
\end{tabular}

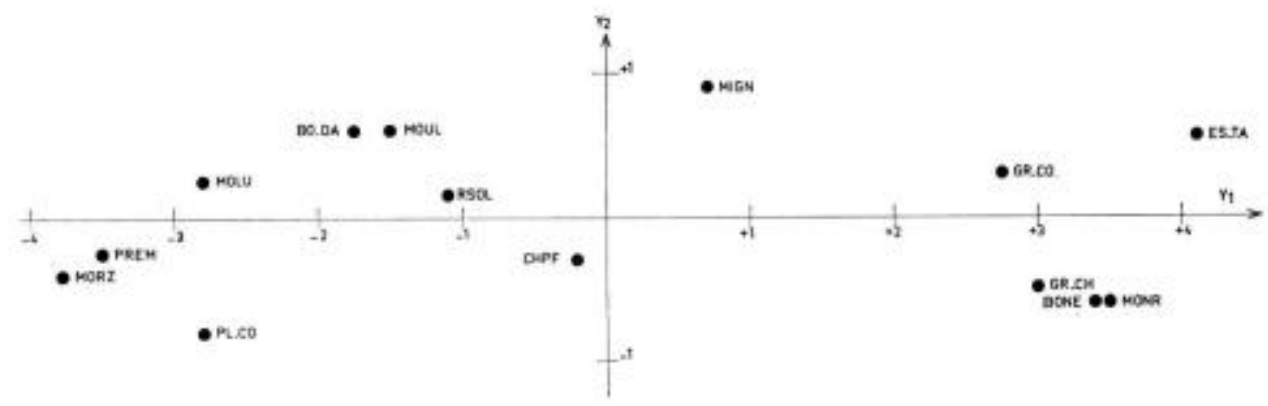

FIG. 7. - Analyse des composantes principales (projection des provenances sur le plan des deux vecteurs propres)

F16. 7. - Principal componenss analysis (projection of provenances on the $t_{m}$ o proper vectors plan) 
Le comportement original de la provenance Gérardmer déjà signalé et l'examen de la figure 7 nous ont conduit à retenir la classification suivante :

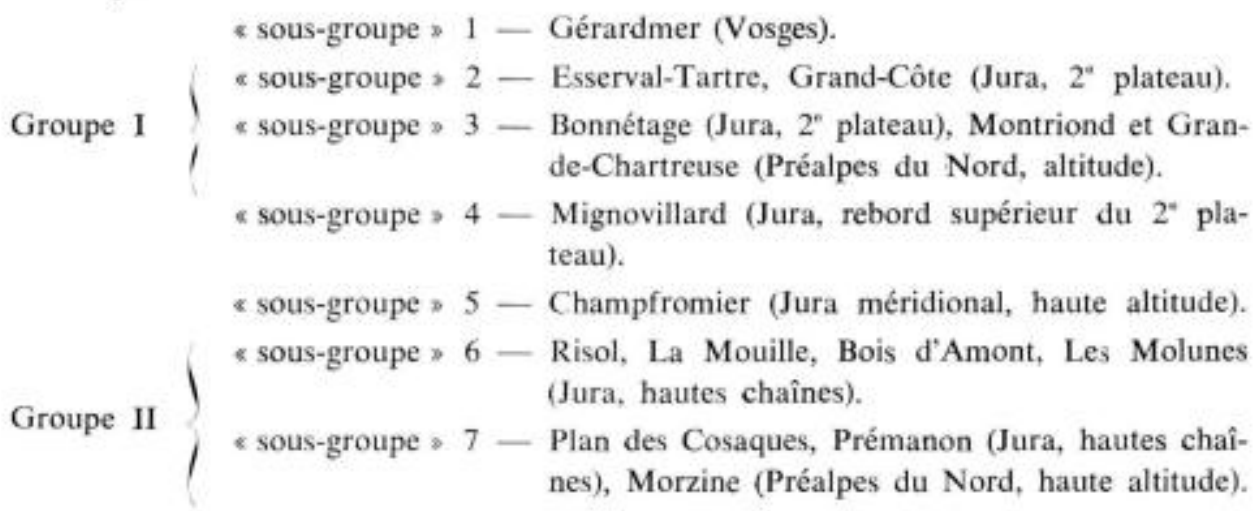

En effectuant les grands regroupements (I et II), nous avons volontairement ignoré les provenances isolées, très vigoureuses comme Gérardmer (Vosges) ou intermédiaires comme Mignovillard (Jura, rebord supérieur du $2^{*}$ plateau, faisant transition entre celui-ci et les hautes chaînes) et Champfromier (Jura méridional, haute altitude).

\section{6. - ESTIMATION DE L'HÉRITABILITÉ}

\section{1. - Méthode de calcul}

L'héritabilité est comprise ici comme étant la part de la variance génétique additive dans la variance totale de la population des descendants d'une population mère donnée. Elle s'exprime par la formule $h^{2}=\frac{\sigma_{A}^{2}}{\sigma_{A}^{2}+\sigma^{2}{ }_{w}}$ avec $\sigma^{2} \sigma_{{ }^{A}} \quad \begin{aligned} & \text { Variance génétique additive, } \\ & \sigma^{2} \text { Variance résiduelle. }\end{aligned}$

Les calculs ont été effectués sur les données individuelles grâce au programme UNFAL. passé sur ordinateur IBM 1130 qui effectue lanalyse et la décomposition des variances et covariances à un facteur contrôlé (descendance), en négligeant par conséquent l'effet bloc (1).

Ce programme fournit :

- les moyennes des différents caractères pour chaque descendance,

- les matrices des coefficients de corrélation pour chaque descendance,

- les matrices des coefficients de corrélation inter (entre moyennes de descendance) et intra (entre données individuelles dans les descendances pour l'ensemble des descendances),

- les coefficients de corrélation intra-classe,

- les coefficients de corrélation génétique.

La décomposition des variances dans chaque provenance ou groupe de provenances se fait selon le schéma suivant :

Source de variation ............

$\begin{array}{ccr}\text { DDL } & \text { CM } & \text { Estimation } \\ f-1 & \mathrm{CM}, & \sigma^{2}{ }_{\mathrm{w}}+k r \sigma^{2}, \\ f(k r-1) & \mathrm{CM}_{w} & \sigma^{2}{ }^{2}{ }^{2}\end{array}$

famille ..................

résiduelle .................

totale ....................

$k r f-1$

avec DDL Nombre de degrés de liberté,

CM Carré moyen,

(1) Ce programme a été élaboré et mis au point par la Station de Biométrie du C.N.R.F. 
$\sigma^{2}$, Variance entre familles,

$\sigma^{2}{ }_{u}$ Variance résiduelle (entre individus dans chaque famille).

$r \quad$ Nombre de répétitions (7).

k Nombre d'individus par sous parcelle (16).

Puisqu'il s'agit de familles de demi-frères, $\sigma^{2}$, est une estimation de $1 / 4$ de la variance génétique additive $\sigma^{2}{ }_{\mathrm{A}}=4 \sigma^{2}$.

Le coefficient de corrélation intra-classe $\frac{\sigma^{2},}{\sigma^{2},+\sigma^{2}{ }_{w}}$ fournit donc une estimation du quart de l'héritabilité.

\section{2. - Validité de la méthode}

Il convient d'attirer l'attention sur un certain nombre d'inconvénients de la méthode.

- Les familles maternelles obtenues par pollinisation libre risquent de comporter une certaine proportion de vrais frères, ce qui conduirait ici à une surestimation de la valeur de l'héritabilité.

- Les mesures exploitées concernent un matériel très jeune. Les valeurs obtenues ne pourraient en aucun cas être utilisées pour des épicéas adultes. Par ailleurs, l'effet maternel n'est peut-être pas encore complètement effacé. Par effet maternel, nous entendons surtout la part des différences entre descendances dues à des différences de grosseur de graines causées par des conditions particulières de nutrition ou d'ensoleillement des mères.

- Certaines observations concernent des caractères typiquement qualitatifs (débourrement, angle d'insertion des aiguilles, pousse d'août) qui ont été assimilés dans les calculs à des caractères continus, alors que les échelles de notation employées sont par définition discontinues (seuils).

- Les plants échantillonnés pour les mesures de poids secs et densité n'ont pas été pris au hasard, mais choisis parmi ceux dont la hauteur s'écartait le moins de la hauteur moyenne de leur sous-parcelle unitaire: d'où deux conséquences: une meilleure estimation des valeurs moyennes de familles (maigré les faibles effectifs par famille: 10), mais une réduction notable des variances résiduelles (intra-famille) pour tous les caractères en cause. II en résulte dans ce cas particulier une augmentation anormale des valeurs de l'héritabilité des caractères de vigueur mesurés sur ces plants : les calculs aboutissent effectivement à des résultats aberrants (héritabilité souvent supérieure à 1). Il n’a done pas été jugé utile de présenter des estimations de l'héritabilité des caractères de vigueur (dimension et poids) obtenus à partir des échantillons de plants séchés.

- Le nombre de descendances par provenance est beaucoup trop faible (10), d'où un risque d'erreur d'échantillonnage tant pour les mères que pour les pères feffet pollen non neutre). On sait maintenant que la validité de l'estimation de l'héritabilité dépend beaucoup du nombre de familles en cause. Nous avons tenté datténuer ce défaut en regroupant les familles de plusieurs provenances d'après la classification objective présentée au chapitre précédent.

- Le fait de négliger l'effet bloc bien qu'il soit significatif (pour les caractères de vigueur) contribue à augmenter la variance résiduelle, done à réduire les héritabilités correspondantes. 


\section{3. - Résultats}

Les estimations sont portées dans le tableau ci-dessous:

\begin{tabular}{|c|c|c|c|c|}
\hline Caractêres & $\begin{array}{l}\text { Nombre de } \\
\text { plants par } \\
\text { Samille }\end{array}$ & $\begin{array}{c}\text { Groupe } 1 \\
\text { Provenances } \\
\text { de moyenne } \\
\text { altitude }\end{array}$ & $\begin{array}{c}\text { Groupe II } \\
\text { Provenances } \\
\text { de haute } \\
\text { altitude }\end{array}$ & $\underset{\text { Gếrurdmer }}{\text { Ili }}$ \\
\hline Nombrz de familles ................ & & 50 & 70 & 10 \\
\hline Hauteur à 4 ans ................. & 112 & 0,42 & 0.99 & 0,13 \\
\hline 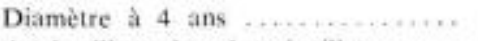 & 112 & 0,12 & 0,40 & 0,08 \\
\hline Angle d'insertion des aiguilles ....... & 112 & 0.05 & 0,48 & 0,08 \\
\hline Pousse daoût, 3* année ........... & 112 & 0.44 & 0,46 & 0,16 \\
\hline Pousse d'août, $4^{*}$ année,$\ldots . . . . . . .$. & 112 & 0,31 & 0,33 & 0.16 \\
\hline $\begin{array}{c}\text { Débourrement } 4 \text { r année (somme des } \\
4 \text { notations) } \ldots \ldots \ldots \ldots \ldots \ldots \ldots\end{array}$ & 112 & 0,51 & 0,33 & 0,28 \\
\hline Densité du bois ...... $\ldots \ldots \ldots \ldots \ldots$ & 10 & 0,26 & 0,47 & 0,12 \\
\hline
\end{tabular}

Groupe 1: Grande-Chartreuse, Bonnétage, Montriond, Grand-Còte, Esserval-Tartre.

Groupe II : Prémanon, Morzine, Plan des Cosaques, Molunes, Bois d'Amont, La Mouille, Risol.

La précision de ces diverse; estimations varie avec le nombre de familles et le nombre de plants par famille utilisé pour les calculs. D'un point de vue statistique, les précisions des valeurs obtenues pour chacun des deux grands groupes sont comparables.

La provenance Gérardmer figure isolément : il est impossible en effet de la rattacher à l'un des deux groupes précédents (population significativement différente de toutes les autres). Nous avons jugé utile de la prendre en considération, malgré limprécision des calculs (10 familles), car cette source de graines est connue et sert fréquemment de référence dans les expérimentations sur l'épicéa.

Compte tenu des écarts entre effectifs de familles, il serait peu logique de comparer les valeurs d’héritabilité de densité du bois à celles des autres caractères.

\section{4. - Discussion}

Ces résultats autorisent les commentaires suivants :

- L'héritabilité de la hauteur est, dans tous les cas, supérieure à celle du diamètre. Ce résultat est logique : on sait en effet que la grosseur des arbres est plus affectée par les actions extérieures (en particulier la concurrence) que la hauteur.

- Les héritabilités des caractéristiques de dimensions sont nettement plus fortes pour les populations peu vigoureuses de haute altitude. Elles atteignent même dans cette expérience des valeurs que l'on peut considérer comme très élevées.

Le groupe de populations de moyenne altitude, à croissance plus rapide, accuse une héritabilité faible pour le diamètre et moyenne pour la hauteur. Ces valeurs tendent à devenir très faibles pour la provenance très vigoureuse Gérardmer.

Comment expliquer ees divergences? 
Une première hypothèse fait intervenir le fait qu'au moment des mesures, ces diverses populations ou groupes de populations avaient le même âge, mais des dimensions moyennes nettement différentes. On peut penser en particulier que la concurrence s'est exercée de manière plus intensive au sein des populations vigoureuses, entraînant une augmentation de la variance phénotypique intrapopulation d'où une réduction " parasite * de la valeur de l'héritabilité. En d'autres termes, il conviendrait de comparer des estimations calculées sur des populations ayant atteint un stade de développement analogue. En fait, il a été souligné précédemment (\$ 33) que les coefficients de variation résiduels des diverses provenances restaient relativement stables pour tous les caractères de vigueur, sauf le diamètre (en particulier pour Gérardmer). Ceci tendrait à prouver que dans les conditions expérimentales décrites, la réduction de la valeur de l'héritabilité, en particulier pour la hauteur, due aux effets de concurrence, serait de faible importance.

Nous suggérons done une deuxième hypothèse. La pression de sélection en haute altitude s'exercerait contre les individus hétérozygotes (très vigoureux) pour des raisons d'adaptation (par exemple élimination des sujets à cime large sensibles aux dégâts de givre), le phénomène étant inversé dans les forêts de moyenne altitude. Le caractère vigueur serait soumis à un contrôle génétique faisant moins intervenir les interactions entre gènes pour les pessières de haute altitude que pour celles de moyenne ou hasse altitude.

L'aptitude à produire des pousses d'août est affectée d'une héritabilité de valeur moyenne dans les deux grands groupes étudiés.

L'héritabilité du débourrement atteint des valeurs moyennes, un peu plus faibles pour le groupe haute altitude. Cela peut surprendre; en fait, la précision des estimations est vraisemblablement biaisée par la méthode d'appréciation du caractère, comme nous l'avons précisé plus haut.

Les valeurs obtenues pour la densité du bois pourraient sembler un peu faibles, ce caractère ayant la réputation d'être fortement héritable. Nous savons que la faiblesse de l'échantillonnage est une cause d'erreur.

Nous devons enfin apporter la précision importante suivante :

Les valeurs d'héritabilité présentées ci-dessus sont des caractéristiques des populations mères de base, mais elles ont été calculées à partir de mesures effectuées sur des descendants, élevés en milieu relativement homogène. Elles ne seraient d'aucune utilité pour un calcul de prévision de gain génétique fait à partir de mesures effectuées sur les arbres adultes de cette population de base. A cela deux raisons principales: le décalage d’âge entre parents et enfants et surtout le fait que les variations de milieu en forêt sont plus importantes et moins bien contrôlées qu'en pépinière. Il est donc souhaitable de distinguer * l'héritabilité en forêt * qui peut être obtenue par un calcul de régression parents-enfants, de \& l'héritabilité en plantation comparative s, la deuxième étant normalement supérieure à la première dans le cas d'un caractère dont l'expression est fortement influencée par le milieu (vigueur).

En conclusion, nous observons que la notion d'héritabilité, appliquée à une espèce forestière comme l'épicéa, doit être utilisée avec précaution. II s'agit d'un paramètre soumis à de nombreuses causes de variation. Les estimations que l'on peut en faire 
dépendent en particulier des conditions expérimentales du stade de développement et de la population de base étudiée.

\section{7. - CORRÉLATIONS}

\section{1. - Méthode de calcul}

additives coefficient de corrélation génétique exprime la liaison entre les valeurs génétiques

$$
\text { Il s'exprime par la formule } r_{\mathrm{A}}=\frac{\operatorname{COV} \mathrm{X}_{\mathrm{A}} \mathrm{Y}_{\mathrm{A}}}{\sqrt{\sigma_{\mathrm{AX}}^{2} \times \sigma^{2}{ }_{\mathrm{AY}}}}
$$

$\mathrm{X}_{\mathrm{A}}$ Valeurs génétiques additives pour le premier caractère $\mathrm{X}$.

$\mathrm{Y}_{\hat{A}}$ Valeurs génétiques additives pour le deuxième caractère $\mathrm{Y}$.

$\operatorname{COV} \mathrm{X}_{\mathrm{A}} \mathrm{Y}_{\mathrm{A}}$ : Covariance entre les valeurs génétiques additives des caractères $\mathrm{X}$ et $\mathrm{Y}$.

$\sigma_{A X}^{2}$ et $\sigma_{A Y}^{2}$ : Variances génétiques additives des deux caractères.

Le numérateur est obtenu par décomposition des covariances effectuées de la même manière que la décomposition de variance exposée au paragraphe précédent.

\begin{tabular}{|c|c|c|c|}
\hline $\begin{array}{l}\text { Source de variation } \\
\text { amille } \ldots \ldots \ldots \ldots \ldots \\
\text { siduelle } \ldots \ldots \ldots \ldots \ldots\end{array}$ & $\begin{array}{c}\text { DDL } \\
f-1 \\
f(k r-1) \\
k r f-1\end{array}$ & $\begin{array}{l}\mathrm{PM} \\
\mathrm{PM}_{t} \\
\mathrm{PM}_{\text {te }}\end{array}$ & \begin{tabular}{l}
\multicolumn{1}{c}{ Estimations } \\
$\operatorname{cov}_{w}\left({ }_{\mathrm{Yx}}\right)+k r \operatorname{cov} f\left({ }_{\mathrm{Xx}}\right)$ \\
$\operatorname{cov}_{\mathrm{in}}\left(\mathrm{rx}_{\mathrm{rx}}\right)$
\end{tabular} \\
\hline
\end{tabular}

avec DDL Degrés de liberté,

PM Produit moyen,

$\operatorname{COV}_{\mathrm{w}}\left(\mathrm{x}_{\mathrm{x}}\right)$ Part résiduelle do la covariance $\mathrm{xy}$.

$\operatorname{cov} f\left(f_{\mathrm{XY}}\right)$ Part famille de la covariance $\mathrm{xy}$.

$k r \quad$ Nombre d'individus par famille.

Dans le cas de demi-frères, $\operatorname{COV} \mathrm{X}_{\mathrm{A}} \mathrm{Y}_{\mathrm{A}}=4 \operatorname{COV}, \mathrm{XY}$.

Les variances additives sont estimées comme précédemment:

$\sigma^{2}{ }_{\mathrm{AX}}=4 \sigma^{2} r \mathrm{X}$,

$\sigma^{2} \mathrm{AY}=4 \sigma^{2} \mathrm{~F}$.

Nous en déduisons:

$r_{\mathrm{A}}=\frac{\operatorname{COV}_{\mathrm{f}} \mathrm{XY}}{\sqrt{\sigma_{\mathrm{f}}^{2} \times \sigma^{2} r \mathrm{Y}}} \quad$ tous les éléments étant tirés aisément des analyses

- Le coefficien de corrélation phénotypique $\left(r_{\mathrm{P}}\right)$ exprime la liaison entre les valeurs phénotypiques individuelles. II s'exprime par la formule

$$
r_{\mathrm{V}}=\frac{\operatorname{COV}_{\mathrm{w}} \mathrm{xY}+\operatorname{COV}_{\mathrm{r}} \mathrm{xY}}{\sqrt{\left(\sigma^{2}{ }_{w \mathrm{X}}+\sigma^{2} r \mathrm{X}\right)\left(\sigma^{2}{ }_{w Y}+\sigma^{2} r \mathrm{Y}\right)}}
$$

avec $\sigma^{2}{ }_{u \mathbf{X}}$ Variance résiduelle de $\mathrm{X}$,

$\sigma^{2}{ }^{2} \mathrm{X}$ Y Variance résiduelle de $\mathrm{Y}$,

$\sigma^{2}+\mathrm{X}$ Variance familles de $X$,

$\sigma^{2} r$ Variance familles de $\mathrm{Y}$.

Ces différentes valeurs sont obtenues à partir du programme UNFAL:

$$
\operatorname{Cov}_{s}\left({ }_{\mathrm{xy}}\right)=r_{\text {intra }} \quad \sqrt{\mathrm{CM}_{\mathrm{r}}(\mathrm{Y}) \times \mathrm{CM}_{w}(\mathrm{x})}
$$

avec $r_{\text {intra }}$ Coefficient de corrélation intra-descendance,

$\mathrm{CM}_{w}(\mathrm{X})$ Carré moyen résiduel de $\mathrm{X}$,

$\left.\mathrm{CM}_{w}{ }_{\mathrm{w}}\right)$ Carré moyen résiduel de $\mathrm{Y}$. 


$$
\operatorname{Cov}_{f}(\mathrm{xr})=r_{\mathrm{A}} \quad \sqrt{\sigma^{2} r \mathrm{x} \times \sigma^{2} r y}
$$

avec $r_{\mathrm{A}} \quad$ Coefficient de corrélation génétique.

Le coefficient de la corrélation due à l'environnement $\left(r_{r}\right)$ exprime ici les liaisons dues au milieu et aux effets génétiques non additifs (dominance, épistasie, etc...). Il se déduit de l'équation classique: $r_{\mathrm{P}}=h_{\mathrm{X}} h_{\mathrm{Y}} r_{\mathrm{A}}+e_{\mathrm{X}} e_{\mathrm{Y}} r_{\mathrm{ce}}$

avec $r_{e w}$ Coefficient de corrélation dû à l'environnement,

$h_{\mathrm{x}}$ racine carrée de l'héritabilité de $\mathrm{X}$,

$h_{Y}$ racine carrée de l'héritabilité de $\mathrm{Y}$,

$e_{\mathrm{X}}=\sqrt{1-h^{2} \mathrm{x}}$

$e_{Y}=\sqrt{1-h^{2}{ }_{Y}}$

Nous en déduisons $r_{r v}=\frac{r_{\mathrm{P}}-h_{\mathrm{X}} h_{\mathrm{Y}} r_{\mathrm{A}}}{e_{\mathrm{X}} e_{\mathrm{Y}}}$

\section{2. - Validité de la méthode}

Certaines réserves évoquées à propos des estimations de l'héritabilité s'appliquent également aux estimations des coefficients de corrélation génétique; énumérons-les brièvement :

- Appréciation des caractères qualitatifs par une échelle de notation discontinue.

- Nombre de descendances par provenance trop faible (pour Gérardmer, 8 degrés de liberté seulement).

- Effet bloc négligé.

Par contre, d'autres causes d'erreur signalées pour l'héritabilité ne devraient pas affecter sensiblement les estimations des coefficients de corrélation génétique; il s'agit en particulier de :

- L'utilisation de familles maternelles issues de pollinisation libre: le coefficient de parenté n'intervient pas dans les formules utilisées.

- Le faible effectif de plants échantillonnés pour les mesures de poids secs et densité : les variances résiduelles n'interviennent plus.

Les estimations des coefficients de corrélation phénotypique sont nettement moins sûres, compte tenu du nombre d'éléments estimés utilisés dans les calculs.

Celles des coefficients de corrélation due à l'environnement sont encore plus douteuses. Nous remarquerons que la formule choisie fait intervenir de nombreux paramètres eux-mêmes peu précis (héritabilité et corrélations phénotypiques).

Pour ces raisons, les deux derniers coefficients de corrélation ont été calculés pour un nombre limité de couples de caractères.

\section{3. - Résultats}

Les calculs ont été effectués sur les mêmes groupes de populations que précédemment (héritabilités), à savoir :

Groupe I : Provenances de moyenne altitude: Grand-Côte, Bonnétage, Montriond, Grande-Chartreuse, Esserval-Tartre : 50 familles. 
Groupe II: Provenances de haute altitude: Prémanon, Morzine, Plan des Cosaques, Molunes, Bois d'Amont, La Mouille, Risol : 70 familles.

Groupe * III * : Gérardmer : 10 familles.

Il est rappelé que la précision des résultats obtenus varie :

- suivant le groupe, en fonction du nombre de descendances pris en considération (en particulier la précision des estimations concernant Gérardmer est faible) ;

- suivant les caractères envisagés en fonction du nombre des individus échantillonnés par descendance (10 seulement pour poids secs et densité).

\subsection{Entre mesures de vigueur.}

Les coefficients sont toujours élevés; nous avons choisi quelques couples caractéristiques.

Dimensions et poids de tige. - Le tableau ci-dessous fournit des estimations du coefficient de corrélation génétique $\left(r_{\mathrm{A}}\right)$. Les valeurs de $r_{\mathrm{V}}$, et $r_{\mathrm{ev}}$ ont été calculées pour la seule liaison hauteur-diamètre.

\begin{tabular}{|c|c|c|c|}
\hline Couples & 1 & 11 & III \\
\hline Hauteur 4 ans - Poids de tige .... & 0,72 & 0,97 & 0,81 \\
\hline Diamètre 4 ans - Poids de tige .... & 0,83 & 0,90 & 0,85 \\
\hline Hauteur 4 ans - Diamètre 4 ans ... & $r_{1}=0,75$ & $r_{\mathrm{F}}=0,83$ & 0,66 \\
\hline & $\hat{r}_{\mathrm{A}}=0,81$ & $\mathrm{r}_{\mathrm{A}}=0,93$ & 0,58 \\
\hline Hauteur 4 ans - Hauteur 3 ans .... & $\begin{array}{r}r_{\text {trr }}=0,08 \\
0,93\end{array}$ & $\begin{array}{r}r_{e r}= \\
0,085 \\
0,96\end{array}$ & $\begin{array}{l}0,07 \\
0,85\end{array}$ \\
\hline
\end{tabular}

Les coefficients concernant les populations de moyenne altitude semblent légèrement inférieurs. On peut supposer qu'en présence d'une concurrence accentuée, les estimations des moyennes de familles subissent des perturbations plus accusées pour le diamètre que pour les hauteurs. On ne peut pas écarter non plus l'hypothèse suivante : le contrôle génétique du diamètre comporterait une part additive moins grande que celui de la hauteur, et le phénomène serait plus accusé chez les provenances vigoureuses.

Les liaisons phénotypiques entre hauteur et diamètre s'écartent peu des liaisons génétiques; la corrélation due à l'environnement et aux effets génétiques non additifs est négligeable pour ce couple de caractères, sauf dans le cas de Gérardmer.

On note également l'excellente liaison entre les deux hauteurs mesurées à un an d'intervalle (possibilité d'économies dans les programmes de mesures juvéniles).

Poids d'organes et certains rapports de poids d'organes. - Nous présentons ici, non plus le coefficient de corrélation génétique, mais le coefficient de corrélation entre moyennes de descendances $\left(r_{1}\right)$. Ce coefficient est une approche de la liaison génétique 
dont l'utilisation se justifie, car les variances génétiques au sein des groupes I et III sont biaisées en raison du faible effectif par famille.

$$
r_{\mathrm{I}}=\frac{\mathrm{PM}_{f(\mathrm{xx})}}{\sqrt{\left.\mathrm{CM}_{f}(\mathrm{x}) \times \mathrm{CM}_{f(\mathrm{x}}\right)}}
$$

avec $\quad \mathrm{PM}_{f} \quad$ Produit moyen famille.

$\mathrm{CM}_{f}(\mathrm{x})$ Carré moyen famille pour le caractère $\mathrm{X}$.

$\mathrm{CM}_{f}(\mathrm{y})$ Carré moyen famille pour le caractère $\mathrm{Y}$.

Soit, en se reportant au modèle d'analyse et de décomposition des covariances utilisé (cf. $\S 71$ ):

$$
r_{\mathrm{I}}=\frac{\operatorname{cov}_{\mathrm{w}}(\mathrm{xy})+k r \operatorname{Cov}_{\left.f_{\mathrm{Xx}}\right)}}{\sqrt{\left(\sigma^{2}{ }_{w}(\mathrm{x})+k r \sigma^{2}{ }_{f}(\mathrm{x})\right)\left(\sigma^{2}{ }_{w}(\mathrm{r})+k r \sigma^{2}{ }_{f}(\mathrm{v})\right)}}
$$

car $\quad \mathrm{PM}_{f}(\mathrm{xy})=\operatorname{COV}_{\mathrm{s}}(\mathrm{xy})+k r \mathrm{COV}_{/}\left(\mathrm{xx}_{\mathrm{xy}}\right)$

et $\quad \mathrm{CM}_{f}(\mathrm{x})=\sigma^{2}{ }_{\mathrm{i}}(\mathrm{x})+k r \sigma^{2}{ }_{f}(\mathrm{x})$

$\mathrm{CM}_{f(\mathrm{r})}=\sigma^{2}{ }_{\mathrm{u}}(\mathrm{Y})+k r \sigma^{2}{ }_{\mathrm{\gamma}}(\mathrm{x})$.

On constate effectivement que cette expression se rapproche d'autant plus de la corrélation génétique que le nombre d'individus par descendance est plus grand (ici, $k r=10$ ) et que les variances et covariances résiduelles sont plus faibles.

Ces coefficients sont dans l'ensemble très élevés, pratiquement toujours supérieurs à 0,70 et significatifs au seuil de probabilité $1 \%$. Le tableau ci-dessous fournit à titre

\begin{tabular}{|c|c|c|c|}
\hline Couples & I & II & III \\
\hline Racines - Tige $\ldots . . . . . . . . . .$. & 0,79 & 0.89 & 0,69 \\
\hline Branches - Tige ................. & 0,67 & 0,96 & 0,86 \\
\hline Aiguilles - Tige $\ldots . . . \ldots \ldots \ldots \ldots . . .$. & 0,79 & 0,97 & 0,90 \\
\hline Poids total - Tige ................. & 0,90 & 0,98 & 0,93 \\
\hline
\end{tabular}
d'exemple les relations entre poids de tige et poids d'autres organes.

Ceci traduit une harmonie fortement imprimée entre les différents éléments de l'arbre.

La légère réduction de l'intensité des liaisons dans les groupes I et III s'explique probablement en tenant compte de l'effet concurrence. En particulier, certaines descendances vigoureuses auraient (proportionnellement) moins de branches parce que la compétition s'y est exercée plus fortement.

Il est possible aussi que la sélection de sujets vigoureux se traduise par une légère réduction (en proportion) du système racinaire, d'où le risque d'une sélection indirecte défavorable à l'adaptation, ce phénomène mériterait une particulière attention pour les populations d'altitude. 
Nous avons également examiné la liaison (au niveau moyennes de descendances) entre la production sous forme de tige et deux rapports caractéristiques exprimant la répartition de la masse de matière sèche entre divers organes importants:

$$
\begin{aligned}
& \frac{\text { poids sec partie aérienne }}{\text { poids sec racines }}=Q 1 \\
& \frac{\text { poids sec aiguilles }}{\text { poids sec total }}=Q_{2}
\end{aligned}
$$

\begin{tabular}{|c|c|c|c|}
\hline Couples & I & II & III \\
\hline $\begin{array}{l}\text { Tige }-Q^{1} \ldots \ldots \ldots \ldots \ldots \ldots \ldots \ldots \\
\text { Tige }-Q_{2}^{2} \ldots \ldots \ldots \ldots \ldots \ldots \ldots \ldots\end{array}$ & $\begin{array}{r}+0,19 \\
-0,09\end{array}$ & $\begin{array}{l}+0,82 \\
+0,56\end{array}$ & $\begin{array}{l}+0,51 \\
+0,20\end{array}$ \\
\hline
\end{tabular}

L'hypothèse précédente est confirmée ; des individus vigoureux des populations II produiraient proportionnellement moins de racines, le phénomène étant moins accusé apparemment dans les populations à croissance plus rapide.

Dans les populations de haute altitude, l'individu vigoureux bénéficierait d'une aptitude génétique à produire une masse d'aiguilles proportionnellement plus élevée. Pour les provenances de moyenne altitude, il faudrait chercher une autre explication à la vigueur, comme par exemple un meilleur rendement photosynthétique (BARADAT, 1967).

\subsection{Entre mesures de vigueur et date de débourrement.}

Ces deux catégories de caractères sont fréquemment associées dans les programmes de sélection individuelle (sélection pour croissance et tardiveté), ce qui explique l'importance attachée aux relations qu'ils pourraient avoir entre eux. Pour les appréhender, nous avons cherché à isoler les trois types de liaisons discernables: phénotypique $\left(r_{\mathrm{P}}\right)$, génétique additive $\left(r_{\mathrm{A}}\right)$, due à l'environnement $\left(r_{\mathrm{eq}}\right)$ dans les deux grands groupes

\begin{tabular}{|c|c|c|c|c|c|c|c|}
\hline \multirow{2}{*}{ Couples } & \multicolumn{3}{|c|}{ I } & \multicolumn{3}{|c|}{ II } & \multirow{2}{*}{$\begin{array}{l}\text { III } \\
r_{A}\end{array}$} \\
\hline & $r_{1}$ & ${ }^{r_{A}}$ & $r_{e r r}$ & $r_{P}$ & $r_{A}$ & $r_{e r}$ & \\
\hline $\begin{array}{l}\text { Diamètre } 4 \text { ans - Somme } \\
\text { des notes de débourre- } \\
\text { ment, } 4^{\prime} \text { année ......... }\end{array}$ & $-0,27$ & $+0,19$ & $-0,49$ & $-0,23$ & $-0,38$ & $-0,14$ & $-0,77$ \\
\hline $\begin{array}{l}\text { Hauteur } 4 \text { ans - Somme } \\
\text { des notes de debourre- } \\
\text { ment } 4^{*} \text { année }\end{array}$ & $-0,27$ & $-0,08$ & $-0,43$ & $-0,26$ & $-0,39$ & $-0,18$ & $-0,01$ \\
\hline
\end{tabular}
de populations retenues.

Une première constatation siimpose: les liaisons génétiques additives diffèrent notablement entre les groupes I et II. 
Dans les populations de haute altitude, vigueur et précocité du débourrement se relient négativement. Dans le groupe I, au contraire, les deux caractères semblent indépendants.

On remarque également que les corrélations dues à l'environnement qui recouvrent ici - comme précisé ci-dessus - les liaisons dues à des mécanismes génétiques non additifs, sont plus fortes dans le groupe I que dans le groupe II.

L'explication fournie dans le paragraphe 61 semble confirmée: nous avons émis l'hypothèse d'un contrôle génétique additif de la vigueur plus marqué dans le groupe II que dans le groupe I, alors que le mécanisme de transmission du débourrement varierait peu entre ces deux groupes. Il est logique que, pour ce couple de caractères, la corrélation génétique additive diminue et la corrélation due à des effets génétiques non additifs augmente dans les populations à croissance plus rapide.

Ces résultats montrent également qu'il peut exister des différences appréciables entre coefficients de corrélation phénotypique et génétique additifs, ce dernier seulement présentant un intérêt pour les calculs de prévision de gain.

Enfin, nous constatons que les corrélations dues à l'environnement (au sens élargi déjà défini) expriment une liaison toujours négative entre précocité et vigueur. Ceci confirme un point de vue déjà exprimé (LACAZE, 1969). Le bourgeon terminal, chez l'épicéa, débourrerait d'autant plus tardivement que sa distance au sol est plus grande, done que l'arbre est plus grand, même si l'écart de hauteur est seulement dû à l'action du milieu.

\subsection{Entre mesures de vigueur et notations de pousse d'aồt.}

Les valeurs des coefficients de corrélation génétique sont portées dans le tableau ci-dessous :

\begin{tabular}{|c|c|c|c|}
\hline Couples & 1 & II & III \\
\hline Hauteur 3 ans - Pousse d'août, $3^{\circ}$ année & 0,74 & 0,77 & $-0,13$ \\
\hline Hauteur 4 ans - Pousse d'aoôt, $4^{\circ}$ année & 0,39 & 0,79 & 0,053 \\
\hline Hauteur 4 ans - Pousse daoût, $3^{*}$ année & 0,82 & 0,82 & 0,53 \\
\hline $\begin{array}{c}\text { Diamètre } 4 \text { ans }- \text { Pousse d'août, } 4^{\prime} \text { an- } \\
\text { née }\end{array}$ & 0,21 & 0,68 & -0.27 \\
\hline 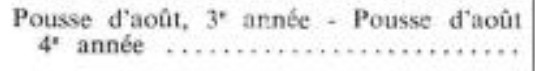 & 0,43 & 0.77 & 0,95 \\
\hline
\end{tabular}

L'aptitude d'une descendance à produire des pousses d'août permet de diagnostiquer la vigueur de la mère.

Il apparaît une fois de plus une divergence entre les deux grands groupes. La liaison génétique reste étroite au cours des deux années d'observation pour le groupe II ; par contre, la relation faisant intervenir les notations de $4^{n}$ année est moins nette dans le groupe I, et encore moins pour la population Gérardmer.

Il serait hasardeux d'avancer une explication, sauf peut-être celle-ci qui mériterait d'être confirmée expérimentalement: au sein des populations très vigoureuses, et en 
présence de concurrence, certains individus parmi les plus vigoureux ont une durée d'élongation prolongée sans $2^{\circ}$ pousse, alors qu'au contraire le rythme d'élongation de sujets dominés comporterait un arrêt précoce suivi d'une deuxième élongation peu importante.

\subsection{Entre vigueur et couleur.}

\begin{tabular}{|c|c|c|c|}
\hline Couples & I & II & III \\
\hline 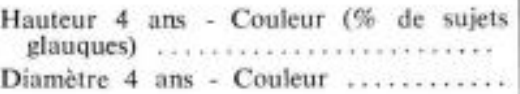 & $\begin{array}{l}-0,26 \\
-0,26\end{array}$ & $\begin{array}{l}-0,32 \\
-0,23\end{array}$ & $\begin{array}{r}-0,64 \\
0,09\end{array}$ \\
\hline
\end{tabular}

La sélection de sujets glauques entraînerait une réduction de la vigueur. La nature de la glaucescence n'a pas été étudiée, mais on peut penser qu'elle correspond à une adaptation à des conditions de milieu difficiles, d'où une nouvelle incompatibilité entre sélection pour la vigueur et adaptation.

Il n'est pas impossible que des caractères de ce type jouent un rôle déterminant dans l'évolution des peuplements d'épicéa d'altitude. L'effet négatif sur la vigueur, exercé par la sélection naturelle en montagne, est confirmé par de nombreuses expériences de provenances; cet effet résulte peut-être indirectement d'une sélection directe pour des critères comme la glaucescence, lui-même lié négativement à la potentialité de croissance.

7.35. Entre vigueur et densité du bois.

\begin{tabular}{c|c|c|c}
\hline \hline Couples & I & II & III \\
\hline & & & $-0,54$ \\
Hauteur 4 ans - Densité du bois ...... & $-0,29$ & $-0,16$ \\
Diamètre 4 ans - Densité du bois .... & $-0,48$ & $-0,60$ & $-0,52$ \\
\hline
\end{tabular}

Les valeurs figurant sur le tableau ci-dessus sont des coefficients de corrélation entre moyennes de familles (voir $\$ 312$ ).

Comme il fallait s'y attendre, les liaisons sont négatives. Ce résultat est classique ; il signifie que chez l'épicéa la sélection en faveur de sujets à croissance rapide se traduit automatiquement par une réduction de la densité du bois produit, donc de ses qualités mécaniques et ceci indépendamment des conditions de milieu.

Le fait que la relation soit plus rigide pour les populations du groupe II s'explique à partir de l'hypothèse proposée dans le paragraphe consacré à la liaison vigueur/ débourrement (part additive plus importante du contrôle génétique de la vigueur dans le groupe II). 
On note aussi, et c'est logique, que les mesures de diamètre interviennent plus fortement que celles de hauteur pour expliquer la densité.

En toutes hypothèses, il s'agit là d’un élément défavorable, mais dont il faut tenir compte dans les programmes d'amélioration de l'épicéa.

Ces quelques résultats, malgrế leur imprécision, tendent à prouver que les corrélations génétiques, comme les héritabilités, sont des paramètres caractéristiques des populations et non pas de l'espèce.

\section{8. - CONCLUSION. \\ CONSÉQUENCES POUR LA SÉLECTION INDIVIDUELLE}

Les résultats obtenus dans les conditions de cette expérience se récapitulent comme suit :

\section{Populations de haute altitude}
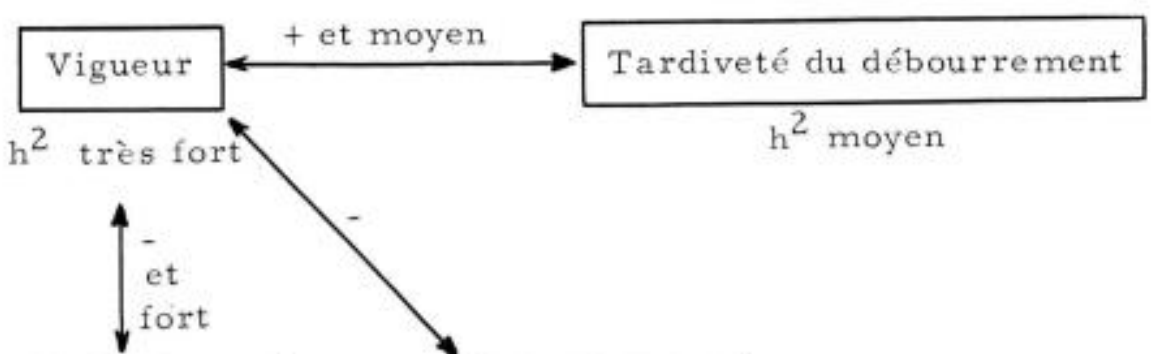

\section{Densité du bois}

$h^{2}$ moyen

Adaptation

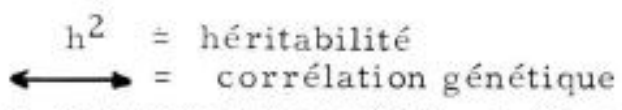

échelle approximative : $0,75-1,00=$ très fort

$0,50-0,75=$ fort

$0,25-0,50=$ moyen

$0,00-0,25=$ faible.

Nous serions donc ici dans une situation idéale pour une sélection en faveur de deux critères vigueur et tardiveté. En fait, dans ce type de population, le critère essentiel est celui de la survie en conditions difficiles, ce qui devrait se traduire par une 
certaine réduction de la vigueur. Il convient de souligner le risque que présenterait ici une sélection — même inconsciente — d'individus à croissance rapide.

\section{Populations de moyenne altitude}

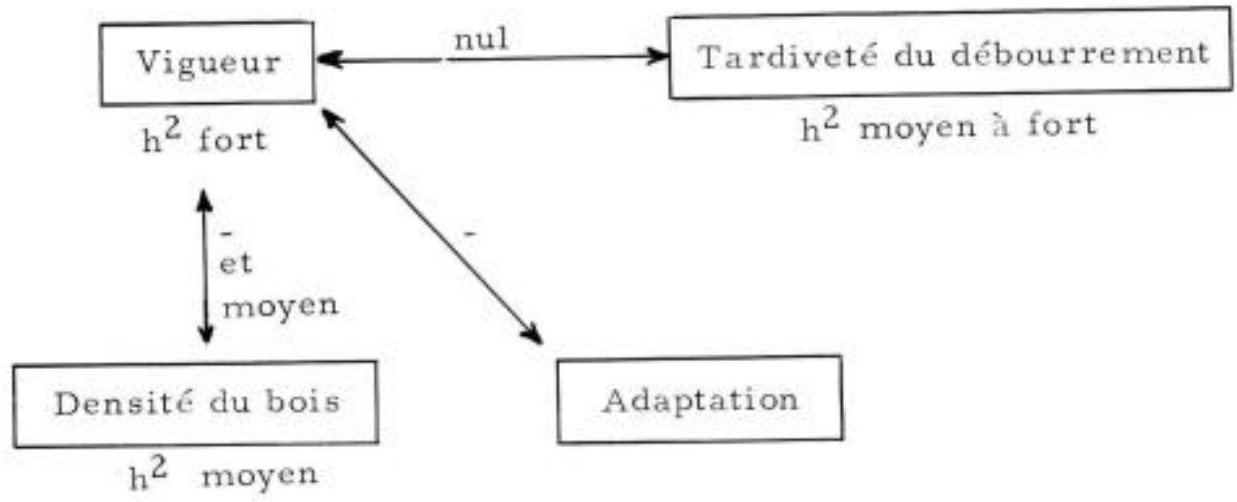

Les trois critères principaux: vigueur, densité du bois et tardiveté du débourrement, retenus en particulier pour la création de variétés utilisables à basse altitude, ont des héritabilités suffisamment élevées pour justifier la sélection individuelle (sur plantation comparative, comme précisé précédemment).

Leurs relations génétiques se présentent sous un angle moins favorable. Une sélection pour la vigueur ne contribuera pas à améliorer la tardiveté et surtout il existe une certaine incompatibilité entre vigueur et densité. On sait que la situation est heureusement inverse pour d'autres espèces comme le pin maritime.

Cet état de fait conduit à la nécessité d'utiliser des indices de sélection affectant à chaque caractère un poids économique et tenant compte de la liaison défavorable enregistrée. On peut aussi tenter de repérer des individus exceptionnels échappant à cette régression, c'est-à-dire vigoureux et produisant du bois dense. Cette dernière solution semble a priori peu réaliste, l'effectif des mères testées dans une plantation comparative étant obligatoirement limité (1).

Pour des variétés destinées à la moyenne altitude, où les conditions écologiques deviennent plus difficiles, certains critères d'adaptation pourraient devenir importants (exemple: pousse d'août, gelées précoces).

Ces réflexions montrent la complexité des problèmes relatifs à la sélection individuelle qui ne peut être logiquement engagée sans étude préalable. La sélection de populations (provenances) garde done tout son intérêt parce que beaucoup plus simple et efficace au stade actuel de nos connaissances.

Reçu pour publication en mars 1971.

(1) La sélection en forêt permettrait de travailler sur des effectifs plus importants mais les valeurs d'héritabilité à prendre en considération seraient vraisemblablement fort différentes. 


\title{
REMERCIEMENTS
}

Les calculs ont été exécutés par la Station de Biométrie du C.N.R.F. sous la direction de C. Miller (Chargé de Recherches) dont les conseils éclairés nous ont beaucoup aidés.

Les mesures de densité ont été effectuées par la Station de Recherches sur la Qualité des Bois, et les résultats obligeamment communiqués par H. Polge (Directeur de Recherches).

Qu'ils trouvent ici l'expression de nos très sincères remerciements.

\section{SUMMARY}

\author{
INFRASPECIFIC VARIABILITY OF NORWAY SPRUCE (\& PICEA ABIES \& KARST., \\ NORTHERN PART OF THE FRENCH AREA). HERITABILITIES \\ AND GENETIC CORRELATIONS OF SEVERAL CHARACTERS (EARLY STAGE)
}

This paper reports an experiment devoted to 15 natural spruce (Picéa abies Karst) provenances sampled in the northern part of the french area of this species. Each provenance is represented by 10 maternal progenies. Measures and notations concerned young plants ( 3 and 4 years old).

The between provenances variability results corroborate those from former experiments, that is to say:

- Vosges (Gérardmer) : good growth, early flushing, numerous lamma shoots, low percentage of trees with glaucous needles, low wood density.

- 2nd plateau Jura and prealps (altitude) : medium vigor, late flushing (except Mignovillard), average percentage of trees with lamma shoots, low percentage of glaucous individuals, average wood density.

- High chains Jura, prealps (high altitude) : low vigor, early flushing, scarce lamma shoots, high percentage of glaucous needles individuals, high wood density.

Two aspects to the wishin provenances variability is studied: between families and within families (residual).

The between families variability expesses, with several reserves, the additive genetic variance of mother trees. Its value is rather important for the chosen characters (sizes, and phenology). It seems that the use of several \& plus s trees progenies does not introduce any bias ; on the contrary, the number of mother trees per provenance (10) is obviously too short (sampling error deviation to normal distribution). The amplitude of between family variability notably differs with provenances. For growth, it reachs the highest level within high altitude provenances.

The within families variability expresses various effects [genetic (additive or not) and environmental effects]. This variance may also differ between provenances, but those deviations are mainly due to a scale effect.

The di-tribution of those various kinds of variability between different levels shows that :

- For height, the within families variance (impossible to use) represents nearly half the total variance. The part of variability due to provenance effect $(35 \%)$ is here more important than the percentage due to individual variability (mothers) $(4 \times 5,6 \%)$.

- For flushing. the residual variance is still more important $(78 \%)$ but individuals (mothers) variance exceeds provenances variance.

This means that, in breeding programs, provenances selection is a good way to improve for growth, and individual selection must be prefered when flushing is concerned.

An objective classification (principal components analysis) of provenances justifies the following regrouping:

- Group I: 5 altitude provenances from the 2nd Jura plateau and Prealps,

- Group II : 7 high altitude provenances from Jura and Prealps,

- Gérardmer provenance, Vosges - mean altitude.

Within groups $\mathrm{I}$ and $\mathrm{II}$, differences between provenances are small enough to consider that the corresponding families belong to two theoritical and homogeneous populations. This permits to reduce the sampling error due the too small number of mothers per provenance. 
Heritabilities of various characters have been calculated for three entities so defined. Heritability of vigor is notably higher for group II than for group I and Gerardmer. It is supposed than genes interactions take a less important part in high altitude forests (lower level of heterozygoty). Values of heritability of phenological characters are nearly the same for the two groups.

Genetic (additive) correlations between size or weight measures are high. Vigor and lamma shoots occurence are positively linked, vigor and glaucousness negatively. The relation between vigor and early flushing is either negative (group II) or null (group D. The environmental correlation coefficients are negative in each case.

Those results tend to prove that heritability and genetic correlation are parameters which are not characteristies of species but of populations; breeding programs based on individual selection must be elaborated at the level provenance or group of provenances.

\section{ZUSAMMENFASSUNG}

DIE INFRASPEZIFISCHE VARIABILITAT DER FICHTE (\& PICEA ABIES \$ KARST. IM NÖRDLICHEN TEIL DES FRANZÖSISCHEN VERBREITUNGSGEBIETES). HERITABILITÄT UND GENETISCIIE KORRELATIONEN EINIGER MERKMALE IM JUGENDSTADIUM.

Die vorliegende Arbeit betrifft die abschliessenden Ergebnisse ciner Versuchsreihe mit fünfzehn natürlichen Herkünften aus dem nördlichen Teil des französischen Verbreitungsgebietes der Fichte (Picea abies Karst.) ; jede Herkunft war durch zehn mütterliche Nachkommenschaften vertreten. Die Beobachtungen und Messungen wurden an drei - bis vier jährigen Pflanzen vorgenommen.

Die Ergebnisse bezïglich der Variabilität zwischen den Herkünften bestätigen jene früherer Versuche und können wie folgt zusammengefasst werden :

-Vogesen (Gérardmer) : hohe Wuchskraft (vigueur), betont frütreibend, hohe Häufigkeit der Johannistriebbildung, geringer Anteil an Pflanzen mit blaugruinen Nadeln, geringe Rohdichte des Holzes.

- Zweites Juraplateau u. Berglagen der Voralpen: mittlere Wuchskraft, spättreibend (mit Ausnahme von Mignovillard); mittlere Häufigkeit der Johannistriebbildung, verhältnismässig geringer Anteil an Pflanzen mit blaugrinen Nadeln, mittelmässige Rohdichte des Holzes.

- Jura (Hochlagen) u. Hochlagen der Voralpen: geringe Wuchskraft, frühtreibend, geringe Häufigkeit der Johannistriebbildung, sehr geringer Anteil an Pflanzen mit blaugrünen Nadeln, hohe Rohdichte des Holzes.

Die Variabilität innerhalb der Herkïnfte wird nach zwei Gesichtspunkten (zwischen den Familien und innerhalb der Familien oder Restvariabilität) untersucht.

Die Variabilität zwischen den Familien bringt mit einigen Vorbehalten die additive genetische Varianz der Muterbăume zum Ausdruck. Sie erreicht bei einigen gemessenen Merkmalen (Wuchskraft und Phänologie) ein sehr hohes Niveau. Die Einbezichung einiger Nachkommenschaften von Plusbäumen scheint keinen systematischen Einfluss zu haben. Im Gegensatz dazu erweist sich die Anzahl der Mutterbäume (zehn) pro Herkunft als deutlich unzureichend (Stichprobenfehler, Abweichung von der Normalverteilung). Die Amplitude der Variabilität zwischen den Familien ist je nach Herkunft sehr verschieden. Beim Merkmal \& Wuchskraft s erreicht sie ihr höchstes Niveau bei den Hochlagenherkünften.

Die Restvariabilität bringt verschiedene Einflüsse zum Ausdruck (additive oder nicht additive genetische Einflüsse, Standortseinflüsse). Die Restvariabilitäten sind gleichfalls je nach Herkunft verschieden, sie können hier jedoch hauptsächlich als ein « Maszstabseffekt s erklärt werden.

Die Untersuchung der Variabilitätsanteile bei den verschiedencn Merkmalen führt zu folgenden Feststellungen :

- Beim Merkmal Höhe beträgt die Restvarianz (unkontrollierbar) ungefähr die Hälfte der Gesamtvarianz. Der Anteil der Variabilität des Faktors Herkunft (35\%) ist in diesem Fall grösser als jener der individuellen Variabilität $(4 \times 5,6 \%)$.

- Beim Merkmal Austreiben ist die Restvarianz noch grösser $(78 \%)$, jedoch scheint die Amplitude der individuellen Variabilität grösser zu sein als jene des Faktors Herkunft. 
Man kann daraus schliessen, dass sowohl die Selektion der Herkünfte zur Verbesserung der Wuchskraft, als auch die individuelle Selektion zur Züchtung spittreibender Pflanzen ihre volle Bedeutung beibehalten.

Eine objektive Klassifizierung der Herkünfte (Prinzipalkomponentenanalyse) ermöglicht die logische Zusammenfassung in folgende Gruppen :

- Gruppe 1:5 Berglagenherkünfte vom zweiten Juraplateau und aus den Voralpen.

- Gruppe II : 7 Hochlagenherkünfte aus der Jura und den Voralpen.

- Herkunft « Gerardmer »: Vogesen, mittlere Höhenlage.

Innerhalb der Gruppen I und II sind diz Unterschiede zwischen den Herkünften verhältnismässig gering, sodass man die Gesamtheit der entsprechenden Familien jeweils als ein Teilkollektiv von zwei theoretisch homogenen Populationen auffassen kann. Der durch die geringe Familienanzahl bedingte Stichprobenfehler kann daher vorteilhaft verringert werden.

Die Heritabilität der verschiedenen Merkmale wurde für dì drei obgenannten Gruppen berechnet. Die Heritabilität der Wuchskraft ist insbesondere bei Gruppe II sehr gross ; es folgen Gruppe 1 und \& Gerardmer \$. Dei Wechselwirkungen zwischen Genen scheinen in den Fichten-Hochlagenbestlinden eine weniger bedeutende Rolle zu spisien (geringeres Hetérozygotieniveau). Die Heritabilität der phänologischen Merkmale ist in den beiden grossen Gruppen annähernd gleich.

Die additiven genetischen Korrelationen zwischen Dimensionen oder Gewichten von Pflanzenorgannen sind sehr hoch. Zwischen Wuchskraft und Johannistriebbildung besteht ein positiver Zusammenhang, während Wuchskraft und blaugrüne Nadelfärbung negativ korreliert sind. Der additive Zusammenhang zwischen Wuchskraft und Frühtreiben ist negativ bei Gruppe II und null bei Gruppe I. Die durch den Standort bedingte Korrelation ist in beiden Fällen negativ.

Die vorleigenden Ergebnisse zeigen deutlich, dass die genetischen Korrelationen und die Heritabilitäten nicht nur artspezifisch sind, sondern charakteristiche Populationsparameter darstellen, und dass die Programme der individuellen Selektion im Rahmen einer Herkunft oder einer Gruppe von Herkünften ausgearbeitet werden müssen.

\section{RÉFÉRENCES BIBLIOGRAPHIQUES}

Baradat Ph., 1967. Elongation de la pousse primaire chez Picea abies Karst., en relation avec la date daoûtement et la teneur en chlorophylle des feuilles. Communication au Groupe d'Eludes des Problèmes de la Physiologie de P'Arbre, Paris, 17 novembre 1967.

Becker W. A., 1967. Manual of procedures in quantitative genetics. 2r édition, Washington State University Press, $130 \mathrm{p}$.

Bouvarel P., 1954. Variabilité de Tépicéa (Pícea excelsa Link.) dans le Jura français, Revue forest. fr. (2), 85-98.

Bouvarel P., 1961. Observations sur la date d'aoûtement de quelques provenances françaises d'épicéa. Ann, Ec. nation, Eaux et Forêts Stn Rech. Expér. forest., 18 (1), 99-129.

Bouvanel P., 1962. L'influence de lorigine des graines d'épicéa sur la croissance en pépinière, la précocité et la fréquence des pousses d'aoút, Ann. Ec, Eaux Forêts, Nancy, 19 (3), 415-439.

Falconer D.S., 1960, Introduction to quantitative genetics, Oliver and Boyd, Edinburgh and London, $365 \mathrm{p}$.

ILly G., 1966. Recherches sur l'amélioration génétique du Pin maritime. Ann. Sci. forest., 23 (4), 780-948. Thèse présentée à I'Université de Bordeaux.

Lacaze J.F., 1969. Etude de la variabilité infraspécifique de l'épicéa (Picéa abiés Karst.). Provenances françaises et polonaises, Résultats au stade juvénile. Ann. Sct, forest, 26 (3). 345-396.

Potge H., Lacaze J. F., 1970. Relations phénotypiques au stade juvénile entre la densité du bois et diverses caractéristiques phénologiques et de vigueur chez Picéa abies Karst., Ann. Sci. forest., 27 (3). 